\title{
Gut Microbiome Composition Differs Extensively Between Indian Indigenous Chicken Breeds Originated in Different Geographical Locations and a Commercial Broiler Line, but Breed-specific as Well as Across-breed Core Microbiomes Are Found
}

Shyam Sundar Paul ( $\nabla$ sspaulcirb@gmail.com )

Indian Council of Agricultural research- Directorate of Poultry Research https://orcid.org/0000-00029549-5741

\section{Rudra Nath Chatterjee}

Indian council of Agricultural Research-Directorate of Poultry Research

Mantena Venkata Lakshmi Narasimha Raju

Indian Council of Agricultural Research

Bhukya Prakash

Indian council of Agricultural Research-Directorate of Poultry Research

Savaram Venkata Rama Rao

Indian Council of Agricultural Research-Directorate of Poultry Research

\section{Satya Pal Yadav}

Indian Council of Agricultural Research-Directorate of Poultry Research

Alagarsamy Kannan

Indian Council of Agricultural Research-Directorate of Poultry Research

\section{Research}

Keywords: Amplicon sequencing, Chickens, Microbiome, Pathogens, 16S rRNA gene

Posted Date: November 25th, 2020

DOl: https://doi.org/10.21203/rs.3.rs-113326/v1

License: (c) (1) This work is licensed under a Creative Commons Attribution 4.0 International License.

Read Full License 


\section{Abstract}

Background: With increasing concern on antimicrobial resistance and given the key role of gut microbiome to the host's nutrition, productivity, development and function of immune system and competitive exclusion of pathogens, characterization of the chicken gut microbiota of different breeds or lines holds high potential for providing clues for developing alternative to antibiotic growth promoters and reducing pathogen load.

Methods: In this study, we analyse and compare the gut microbiome of indigenous Indian Nicobari, Ghagus and Aseel chicken breeds originated in Nicobari island, coastal area and Indian mainland, respectively and a global commercial broiler VenCobb400 line reared without antimicrobials using $16 \mathrm{~S}$ rDNA V3-V4 hypervariable amplicon sequencing.

Results: The dominant gut microbial Phyla were Bacteroidetes and Firmicutes in native breeds whereas in VenCobb 400 the phylum Firmicutes was most dominant. Bacteroidetes/ Firmicutes ratio in Ghagus and Nicobari were similar but differed from that of Aseel. The Aseel, VenCobb 400, Ghagus and Nicobari breed or line presented 3461, 857, 1911 and 1767 distance (97\% cutoff) based operational taxonomic units (OTUs) with $>2$ members. Alpha diversity analysis indicated higher diversity as well as richness in indigenous breeds than in the broiler line. Beta diversity analysis indicated significant difference $(P<0.001)$ in community structure between different breeds or line and clear separation of clusters at different taxonomic levels based on breeds or line. Differential abundance analysis using edgeR detected 88 phylotype OTUs with significant $(P<0.05)$ difference in abundance between groups. Linear discriminant analysis effect size (LEfSe) revealed 82 breed or line specific phylotype OTU level biomarkers. Five phylotype OTUs occurred as core microbiome across all breeds or line representing $13 \%$ of total prokaryote count. Seven phylotype OTUs were core microbiome across the three native breeds representing $49.3 \%$ of total prokaryotic sequences of native breeds. Correlation analysis indicated existence of complex microbial networks in all breeds or line.

Conclusion: The study indicated highest overlap in microbial community composition between the coastal breed (Ghagus) and the island breed (Nicobari) among indigenous breeds and very low overlap between the broiler line and all indigenous breeds. Despite extensive variation, breed specific as well as across breed or line core microbiomes were found. This study provides new insight into the comparative community structure of microbiome of chickens of 4 breeds or line of different genetic background or geographical origin and may facilitate development of breed specific feed additives and alternative to antibiotic growth promoters.

\section{Background}

The chicken is the cornerstone of animal agriculture worldwide with a flock population exceeding 40 billion birds/year [1]. Poultry represents one of the most efficient form of animal protein with highly efficient feed conversion. Ever increasing human population, urbanization and income levels are 
contributing to huge increase in demand for protein and therefore livestock and poultry. Sustainable poultry meat and egg production is important to provide safe and quality protein sources in human nutrition. Feed efficiency and faster growth are the crucial goals in highly competitive poultry production system. For attaining these goals maintaining a healthy gut is an important prerequisite.

The gastrointestinal (GI) tract of chickens is densely populated with diverse and complex microbiota (Bacteria, fungi, Archaea, protozoa, and virus; dominated by Bacteria) that plays a vital role in digestion and absorption of nutrients, host immune system development and pathogen exclusion, endocrine activity, maintaining normal physiological homeostasis and influencing gut development, nutirent supply and host metabolism and detoxification [2]. Understanding diversity and community structure of gut microbiome is important for devising strategy for improving the chicken gut microbiome. Several studies in rats, human, chicken and other species have indicated that gut microbiome composition is strongly influenced by host. Further, host immune pathways in gut tissues may influence microbiome structure. Similarly, some studies have indicated strong correlation between gut microbiome and feed efficiency. In studies with cattle it has been shown that a heritable set of core gut microbiome influence dairy cow productivity [3]. It has been suggested that high throughput sequencing tools have huge potential to be used for assessment of microbiome structure in gut of chicken in a comprehensive manner which in turn may further development of strategy for improving growth, feed efficiency, survivability or lower pathogen shedding via the development of host specific probiotics [4].

Native chicken breeds are gaining popularity across India due to their unique characteristics like desirable flavour of meat and eggs and ability to thrive in low input system and hot humid climate. Further, there is a growing demand for eggs and meat of native chicken in cities as compared to the exotic ones due to their perceived better flavor and taste. Many producers are rearing indigenous chicken breeds under intensive system to meet increasing demand.

Among 17 indigenous chicken breeds of India breeds like Aseel, Nicobari and Ghagus have been shown to have better egg, meat traits, resistance to infectious diseases with better immune parameters but lower feed conversion efficiency as compared to commercial broiler chickens. Analysis to define gut micobiome of native Indian breeds like Asamese chicken [5], Aseel and Kadaknath has been reported recently [6]. But the study on Aseel and Kadaknath was carried out when chickens were supplemented with antibiotic growth promoter. Antibiotics are known to modulate gut microbiota significantly and hence information on true composition of gut microbiota without influence of antibiotics of these breeds remains to be studied.

In the present study we use amplicon sequencing targeting hypervariable (V3-V4) region of 16S rRNA genes to compare gut microbiota of three indigenous Indian breeds originated in diverse geographical regions (Aseel, Ghagus and Nicobari) and a commercial broiler (Vencobb 400) reared under commercial set up but without any antibiotic. The Nicobari breed is an indigenous and endemic breed of chicken of Nicobar Islands which are an internationally acknowledged biodiversity hot spot, off the Indian mainland (got detached from Asian main-lands some 100 million years ago) and produces highest number of eggs 
among all indigenous chicken breeds of India [7] and is believed to have high disease resistance. Aseel is one of the important and most popular indigenous chicken breeds of Indian mainland and is well known for their meat quality with desirable taste and flavor besides ability to thrive under adverse climatic and nutritional conditions [8]. Ghagus is another important native chicken breeds of coastal region of Southern India known for their meat quality with desirable taste and flavor, disease resistance [9] and ability to perform under hot and humid climate and under low plane of nutrition.

Our hypothesis was that the gut microflora would vary significantly between chicken breeds and lines, offering clues for development of breed/line specific probiotics or feed additives for improvement in performance or feed efficiency or health or pathogen exclusion or for targeted genetic improvement by selective breeding for desirable type of gut microbiome.

\section{Methods}

\section{Chicken breeds and experimental design}

In the present study four chicken breeds or lines were chosen for comparison which included three indigenous Indian breeds (Nicobari, Aseel and Ghagus) and one global commercial broiler line (Vencobb 400). The chickens of the indigenous breeds utilized in the present study were collected from ICARdirectorate of Poultry Research farm where these breeds are being conserved separately under intensive system. These birds were originally collected from their respective breeding tracts (Nicobari from Andaman and Nicobar Island, Ghagus from Kolar district of Karntaka State and Aseel from Andhra Pradesh). VenCobb400 line (venkateswara Hatchery and Breeding Farm Pvt Ltd, Hyderabad) were used for comparison as representatives of the global commercial broiler line.

Care was taken to ensure that all birds received exactly the similar husbandry to minimise non-host variation. All chickens were fed maize and soybean-based balanced diet as per respective feeding standards for intensive production system with no antimicrobials having been provided while in production.

\section{Sample collection and DNA extractions}

For each breed/line eight apparently healthy male chickens in their finishing (marketable age) stage (av BW, 2.4 kg; age, indigenous:35 weeks; broiler, 6 weeks) were selected at random from each group, caught and eutha- nized by cervical dislocation. Gut were opened immediately using sterile scissors and the luminal contents of hindgut (from duodenum to cloaca including caeca) were recovered into sterile cryovials, mixed thoroughly and was immediately stored in a portable freezer at $-20^{\circ} \mathrm{C}$, transported to the laboratory and stored at $-80^{\circ} \mathrm{C}$.

Total genomic DNA was extracted from the pooled gut contents of each individual chicken using the commercially available QIAamp Fast DNA Stool Mini kit (QIAGEN, Germany) following the manufacturer's 
Instructions. DNA concentration and quality were assessed using a Qubit 2.0 fluorometer (Invitrogen, ThermoFisher scientific, MA) and gel electrophoresis. DNA was stored at $-20^{\circ} \mathrm{C}$ until further processing.

\section{S rRNA gene amplification and MiSeq sequencing}

Hypervariable V3 and V4 regions within the 16S rRNA gene were amplified from gut microbial DNA sample using the primer pair S-D-Bact-0342-b-S-17(5'-CCTACGGGNGGCWGCAG-3') and S-D-Bact-0785-1A-21(5'-GACTACHVGGGTATCTAATCC-3') recommended by Klindworth et al. [10] with suitable adapters. Each $25 \mu \mathrm{L}$ PCR reaction comprised of $2.5 \mu \mathrm{L}$ DNA ( $5 \mathrm{ng} / \mu \mathrm{L}), 5 \mu \mathrm{L}$ each forward and reverse primer (1 pM) and 12.5 $\mu \mathrm{L} 2$ X KAPA HiFi HotStart ReadyMix (Kapa Biosystems, UK). PCR amplification cycles were as follows, initial denatur- ation at $94^{\circ} \mathrm{C}$ for $3 \mathrm{~min}$, followed by 25 cycles of $95^{\circ} \mathrm{C}$ for $30 \mathrm{~s}, 55^{\circ} \mathrm{C}$ for $30 \mathrm{~s}$ and $72{ }^{\circ} \mathrm{C}$ for $30 \mathrm{~s}$, and a final ex- tension at $72{ }^{\circ} \mathrm{C}$ for $5 \mathrm{~min}$. Amplicons were further proc- essed for library preparation using Illumina's Nextera XT library preparation kit (Illumina, USA). Sequencing was performed using an Illumina MiSeq desktop sequencer. Trim- ming of adaptor sequences was performed using Illumina analysis software V2.5 as recommended by the manufacturer using default parameters.

\section{Sequence data analysis}

Illumina reads were analyzed using MOTHUR software package (v 1.40.0) [11] by following analysis pipeline of Miseq SOP (https://www.mothur.org/wiki/MiSeq_SOP) with some modifications. Briefly, read pairs were assembled into contigs. A threshold of phred quality score $(Q \geq 25)$ of the base were chosen for a stringent quality control processing. Any contigs with ambiguous base $(\mathrm{N})$, having homopolymer bases greater than 8 and shorter than 200 bp were culled. Identical or duplicate sequences were merged. Sequences were aligned to SILVA seed alignment (silva.seed_v138.align ; available in the MOTHUR website). Poorly aligned sequences were removed and overhangs at both ends were trimmed so that they overlap the same region. Unique sequences were screened and further de-noised based on pre-clustered command for up to 2 differences between sequences. Chimera sequences were checked and removed using VSEARCH [12] as implemented in MOTHUR. Sequences were then classified using naïve Bayesian classifier against RDP 16S rRNA gene training set (version 16) with bootstrap cutoff of 51\% [13].

Sequences classified to unrelated taxon were removed. Clean sequences were subjected to operational taxonomic unit (OTU) clustering using DMSC software [14] at 97\% similarity cutoff. DMSC output was converted to MOTHUR formatted list file and shared file for further analysis. OTUs were taxonomically classified using RDP classifier as implemented in mother using GreenGene Taxonomy (gg_13_8_99.gg.tax) database available in the MOTHUR website. MOTHUR formatted shared file and consenus taxonomy files were converted to biom formatted file and singleton and doubleton OTUs were removed from the BIOM file. The BIOM file along with the sample metadata files were uploaded to MetaCoMet web server [15] for plotting Venn diagram of OTUs. The breed specific OTU Tables along with metadata were uploaded to METAGENassist website [16] for analysis where data were filtered as per default settings and samples were normalized using total sum (sample vs. Sample) and Pareto scaling (taxon vs taxon; mean centred and divided by the square root of standard deviation of each variable). The processed data were used for generation of correlation heatmaps using Spearman rank option. The BIOM (or MOTHUR generated shared and consensus taxonomy files) along with the metadata file and a NJ tree 
file prepared from the OTU representatives were uploaded to MicrobiomeAnalyst [17] for analysis of alpha diversity, beta diversity, differential abundance, biomarker identification and correlation network analysis. For the analysis of alpha diversity and beta diversity (nonmetric multidimensional scaling (NMDS) and principal coordinate analysis (PCoA)) data were normalized by cumulative sum scaling (CSS) method after disabling the default data filtering options for low counts and low variances (however, features appearing only in one sample were removed automatically as there was no option to disable such minimal filtering function). For the analysis of differential abundance (using edgeR), biomarker identification (using Linear discriminant analysis effect size or LEfSe) and correlation network data were filtered for low counts and low variances using the default setting to remove less informative features, to focus on important features and to improve downstream statistical analysis (this process removed 127 low count OTUs out of 296 taxonomic OTUs). Beta diversity profiling and significance testing were carried out at different taxonomic levels like OTU, genus, family, class and phylum using PCoA as well as NMDS ordination based on different distance methods like Bray-Curtis dissimilarities, Jensen-Shannon diversion and Weighted Unifrac using statistical methods like permutational multivariate analysis of variance (PERMANOVA) and homogeneity of group dispersion (PERMDISP). Core microbiome analysis were carried out at OTU level where only OTUs with a mean normalized (CSS) relative abundance of at least $0.1 \%$ and having within breed prevalence of at least $50 \%$ in at least one breed were chosen to focus on the most commonly shared OTUs. On detection of significant difference in overall abundance between groups on edgeR analysis followed by Benjamini-Hochberg FDR correction for multiple comparison, groups were compared pairwise using nonparametric Mann Whitney $U$ test (Wilcoxon rank sum test) as implement in SPSS [18]. To identify chicken breed/line-specific biomarkers at multiple taxonomical levels analysis were performed using Linear discriminant analysis (LDA) effect size (LEfSe) algorithm using Benjamini-Hochberg false discovery rate (FDR) adjusted $p$ value cutoff value of 0.05 and the logarithmic LDA score cutoff of 2 as well as 3.5 [19]. The LEfSe bar plots were created using MicrobiomeAnalyst but the cladogram was created using a standalone version of LEfSe [18]. In all analyses $p$ values were corrected for Benjamini-Hochberg false discovery rate (FDR). BIOM data were rarefied to the minimum library size (at 12151 sequences per sample) and rarefaction analysis were carried out using MOTHUR. Rarefaction curve was visualized by creating plots using R package. Alpha diversity matrices were compared at OTU level using Kruskal-Wallis test followed by Dunn's test as implemented in SPSS [18 ] and Benjamini-Hochberg false discovery rate (FDR) adjustment of $p$-values. Correlation networks at different taxonomic levels were built based on the pairwise Spearman rank correlation coefficients where each node represents a taxon and two taxa are connected by an edge if the Spearman rank correlation between the two taxa meet the p-value $(<0.05)$ and correlation $(>0.5)$ thresholds. Taxonomic assignments were presented as Krona charts from CSS normalized relative abundance data using standalone KronaTools (version 2.7.1) [20].

\section{Results}

\section{Microbiome sequencing}


High throughput sequencing generated 5.095 million raw reads corresponding to $4.08 \mathrm{Gbp}$ of raw data from gut content of the 32 chickens. After read quality filtering, merging paired end reads, denoising, removing chimeras and filtering low quality sequences, the average number of quality controlled sequences per sample was 113, 028 (range, 50,392 - 295,955) (Table 1). The 16 s rRNA gene amplicon sequencing results were deposited in the Sequence Read Archive of the NCBI (accession numbers: PRJNA641245 and PRJNA641779).

Table 1

Summary statistics of sequences analysed, OTU numbers detected (clustered at $>97 \%$ similarity) and microbial diversity covered.

\begin{tabular}{|c|c|c|c|c|}
\hline & Aseel & Broiler & Ghagus & Nicobari \\
\hline Total sequences & 2135894 & 493732 & 484707 & 502563 \\
\hline Av. sequences/sample & 266987 & 61717 & 60588 & 62820 \\
\hline Av. No. OTUs $(n>2)$ & 3461 & 857 & 1911 & 1767 \\
\hline Av. Microbial diversity(\% Good's coverage) & 99.1 & 98.6 & 97.2 & 97.7 \\
\hline
\end{tabular}

The indigenous Aseel breed presented the highest number of non-singleton non-doubleton (with $>2$ members) OTUs (genetic distance based OTUs at $97 \%$ similarity curoff) (3461), followed by Ghagus (1911) and Nicobari (1767) (Table 1). The commercial broiler line presented the lowest number of OTUs (857). Observed OTU numbers were significantly $(P<0.05)$ higher in the indigenous breeds than that of the commercial broiler line (Fig. 1 and Additional file 1). Observed OTU numbers in the Aseel breed was higher than those of Ghagus and Nicobari. However, OTU numbers in Ghagus and Nicobari were comparable.

Based on Good's cover- age index, 98.6, 99.1, 97.2 and $97.7 \%$ of gut microbial diversity were covered in Broiler, Aseel, Ghagus and Nicobari, respectively (Table 1).

\section{Taxonomy Assignment}

The assignment of consensus taxonomy resulted in the identification of 18 phyla, 138 genera and 296 phylotype-OTUs (Phylotype-OTUs were obtained after merging distance based OTUs with the same consensus taxonomy) being represented across the gut samples of the chicken population. Figure 1 provides an overview of the average CSS normalized relative abundance levels of most of the abundant microbiota at different taxonomic levels in the gut microbiota of different breeds/line. In Aseel the gut microbiota were dominated by Bacteroidetes (44.0\%) followed by Firmicutes (43.0\%), Proteobacteria (6.0\%), Actinobacteria (1.0\%) and Cyanobacteria (0.8\%) that constituted $94.8 \%$ of the whole phyla. In the broiler line Firmicutes, Bacteroidetes, Cyanobacteria, Proteobacteria and Verrucomicrobia were the major phyla, which accounted for $81.0,6.0,6.0,4.0$ and $1.0 \%$ of total sequences, respectively. In Ghagus 
Bacteroidetes, Firmicutes, Proteobacteria, and Cyanobacteria were the major phyla, which accounted for 62.0, 26.0, 3.0 and $0.9 \%$ of total sequences, respectively. In Nicobari Bacteroidetes (53.0\%), Firmicutes (24.0\%), Proteobacteria (8.0\%), Fusobacteria (5.0\%), Verrucomicrobia(2.0\%) and Cyanobacteria (2.0\%) were predominant phyla. Overall, the two phyla Firmicutes and Bacteroides represented $77-88 \%$ of gut prokaryotes.

In the Broiler line, the Firmicutes phylum consists of predominant genera such as unidentified genus under family Lachnospiraceae (32.0\%), Lactobacillus (18\%), YS2 (6\%), Ruminococcus (5\%), Dorea (4\%), unknown genus under order Clostridiales (4.0\%), Oscillospira (3.0\%), Coprococcus (2.0\%), cc_115 group (2.0\%), Clostridium (1\%), and Blautia (15). Bacteroidetes phylum consists of predominant genus such as Bacteroides (5\%). Proteobacteria phylum consists of predominant genera like an unidentified genus under order RF32 (2.0\%), Escherecia (0.8\%) and Bilophila (0.5\%). The phylum Cyanobacteria is mainly represented by an unknown genus under order $Y S 2$, which constituted $6.0 \%$ of total sequences. The phylum Verrucomicrobia is proportionately less abundant and mainly represented by genus Akkermansia and an unidentified genus under family Cerasicoccaceae.

In Aseel, the Firmicutes phylum consists of predominant genera such as an unidentified genus under family Lachnospiraceae (11.0\%), Oscillospira (6\%), an unknown genus under order Clostridiales (3\%), an unidentified genus under family Ruminococcacae (3.0\%), Coprococcus (3.0\%), Faecalibacterium (2.0\%), Ruminococcus (2.0\%), Dorea (2.0\%), SMB53(2.0\%), unidentified genus under family Christensenellaceae (2.0\%) and Lactobacillus (1.0\%). Bacteroidetes phylum consists of predominant genus such as an unidentified genus under order Bacteroidales (24.0\%), Bacteroides (11.0\%), an unknown genus under phylum Bacteroidetes (4.0\%), an unknown genus under family S24-7 (2.0\%), Paraprevotella (0.8\%) and Prevotella (0.6\%). Proteobacteria phylum consists of predominant genera like Anaerobiospirillum, Sutterella, Desulfovibrio, Escherecia representing 2.0,2.0,0.5 and $0.3 \%$ of sequences respectively. The Actinobacteria phylum is mainly represented by a genus Olsenella ( $1 \%$ of sequences).

In Ghagus, the Firmicutes phylum consists of predominant genera such as Faecalibacterium, SMB53, unknown genus under order Clostridiales, an unidentified genus under family Lachnospiraceae, Oscillospira, Megamonas, Ruminococcus, Lactobacillus and Claustridium representing $4.0,4.0,3.0,2.0,2.0,1.0,1.0,1.0$ and $0.5 \%$ of total sequences, respectively. Bacteroidetes phylum consists of predominant genus such as Bacteroides (25.0\%), an unidentified genus under order Bacteroidales (22.0\%), an unidentified genus under family $S 24-7(5.0 \%)$, an unknown genus under phylum Bacteroidetes (2.0\%), Prevotella (2.0\%) and Odoribacter (0.9\%). Proteobacteria phylum consists of predominant genera like Sutterella and Desulfovibrio, representing 2.0 and $0.8 \%$ of sequences respectively.

In Nicobari, the Firmicutes phylum consists of predominant genera such as an unidentified genus under family Lachnospiraceae, an unknown genus under order Clostridiales, Megamonas, Faecalibacterium, Lactobacillus, SMB53,Oscillospira, Coprococcus, Ruminococcus and Clostridium representing 4.0,3.0,3.0,3.0, 2.0,1.0,1.0, 0.9, 0.9 and $0.5 \%$, respectively. Bacteroidetes phylum consists of predominant genera such as Bacteroides, an unidentified genus under order Bacteroidales, an unidentified genus under 
phylum Bacteroidetes, an unidentified genus under family S24-7, Parabacteroides, Paraprevotella, Prevotella, Parabacteroides and Odoribacter representing 28.0, 16.0,2.0,1.0,0.9,0.9,0.8 and $0.9 \%$ of total sequences. Proteobacteria phylum consists of predominant genera like Sutterella and Desulfovibrio, representing 4.0 and $1.0 \%$ of sequences, respectively.

A venn diagram depicting extent of overlap of phylotype-OTUs between different hosts has been presented in Additional file 1. Aseel, Broiler, Ghagus and Nicobari had 263,172,249 and 230 phylotypeOTUs, respectively. Only 27 phylotype-OTUs were specific to any breed or line (Aseel: 25; Broiler: 1; Ghagus: 8; Nicobari: 3), whereas, 35, 89 and 135 phylotype-OTUs were shared between 2, 3 and 4 groups, respectively.

\section{Microbial alpha diversity and data rarefaction}

Different alpha diversity metrices (the diversity within each breed or line) were estimated to assess different aspects of the community structure and the results are presented in Fig. 2 and Additional file 2. The mean observed richness (number of observed OTUs) was highest in the Aseel group followed by Ghagus and Nicobari and lowest in the broiler line. The species richness (or the number of species or OTUs) indices like ACE and Chao1 were higher $(P<0.01)$ in the indigenous breeds than that of the commercial broiler line. Among indigenous breeds Aseel had significantly higher ACE and Chao1 estimates than those of Ghagus or Nicobari.

The diversity (which takes into account both richness and evenness) estimators like Simpson, Shannon and Fisher were also higher $(\mathrm{P}<0.01)$ in the indigenous breeds (Aseel, Ghagus and Nicobari) as compared to the commercial broiler line and these estimators were comparable among the indigenous breeds. Besides these estimators, rarefaction curves based on the Chao1 index were also plotted. The rarefaction curve depicts the correlation between the number of sequences and the number of OTUs and steeper the slope, the higher the diversity [21]. Rarefaction curve also indicated that the broiler line had lower diversity than those of the indigenous breeds (Additional file 3). Rarefaction curve approached asymptotic level for each breed or line, suggesting the availability of sufficient reads to rep- resent each microbiome community.

\section{Microbial beta diversity}

The beta diversity (the partitioning of biological diversity among breeds or along a gradient, e.g., the number of species shared between two breeds or lines) analysis was undertaken to assess the relationship of microbial communities of different breeds/line using different metrices to calculate the dissimilarity/distance matrix, like Bray-Curtis, Jensen-Shannon, unweighted UniFrac and weighted UniFrac.

The correlation between the distance matrix and metadata categories was tested using PERMANOVA, which reports an $R^{2}$ value indicating the proportion of variation explained by this category, and a $P$ value representing the statistical significance [22]. Homogeneity of group dispersions were also tested using PERMDISP. Beta diversity was visualized using nMDS as well as PCoA but due to space limitation only 
plots obtained using nMDS are presented. Results of beta diversity analysis including results of ordination using nMDS or PCOA are presented in Additional file 4.

PERMANOVA tests performed using all beta diversity metrices used in this study showed significant $(\mathrm{P}<$ 0.001) difference in community structure between different breeds/line both at OTU level and at Phylum level (Additional file 4). At OTU level, Jensen Shannon based PERMANOVA analysis had highest Pseudo$F(11.56)$ and $R^{2}(0.553)$ values among all four distance metrices indicating that $55.3 \%$ of microbiota variation is explained by this category (breed) besides a significant $p$-value $(P<0.001)$. The weighted UniFrac based analysis at OTU level showed that breed explained $47.3 \%\left(R^{2}\right)$ of microbial variation (PERMANOVA, pseudo-F 8.4, P < 0.001). At phylum level, Jensen Shannon based PERMANOVA analysis had highest Pseudo- $F(18.54 ; P<0.001)$ and $R^{2}(0.665)$ value among all four distance metrices indicating that $66.5 \%$ of microbiota variation is explained by this category (breed) besides a significant $\mathrm{p}$-value $(\mathrm{P}<$ 0.001). The weighted UniFrac based analysis at phylum level showed that breed explained $52.7 \%\left(R^{2}\right)$ of microbial variation (PERMANOVA, pseudo-F 10.4, $\mathrm{P}<0.001$ ). The beta dispersion values (PERMDISP) were non-significant for all groups in all diversity metrices analysed at OTU or phylum level except in case of unweighted unifrac analysis for phylum data indicating homogeneous dispersion among groups.

Beta diversity plots visualized using ordination methods nMDS at OTU and phylum level using nMDS method of ordination have been presented in Fig. 3 and Additional file 5, respectively. Jaccard index resulted in similar plots in NMDS scaling as that of Bray Curtis distance both at OTU as well as at Phylum Level and hence, plots for Jaccard index have not been presented.

The NMDS scaling based on all five distance metrices showed clear visual separation of breeds/line at OTU level. When Jensen-Shannon or weighted uniFrac distance was used there was high degree of overlap between the indigenous breeds while only minor overlap between broiler and the indigenous breeds was evident at OTU level. When distance metrices like Bray-Curtis or unweighted UniFrac or Jaccard were used for NMDS plotting there was no overlap between the broiler Line and indigenous breeds at OTU level but there were high degree of overlaps among indigenous breeds. At phylum level, there was high degree of overlap between the indigenous breeds but only minor overlap between Broiler and indigenous breeds (Aseel or Nicobari or Ghagus) was observed and extent of overlap between breeds/line varied with the distance metric used. In case of weighted UniFrac distance there was considerable overlap between broiler and Aseel, minor overlap between broiler and Nicobari and no overlap between broiler and Ghagus. While in case of unweighted UniFrac minor overlap between broiler and Nicobari was observed with no overlap between Ghagus and broiler or between Aseel and broiler. In case of Bray -Curtis distance/Jaccard index minor overlap was observed between broiler and Ghagus and no overlap between broiler and Nicobari or between broiler and Aseel. When Jensen -Shannon divergence was used there was minor overlap between broiler and Aseel and no overlap between broiler and Ghagus or broiler and Nicobari. Overall, it is noticeable that even at Phylum level there was very low level of overlap between indigenous breeds and the broiler line indicating almost completely different microbial community compositional distribution pattern between these two categories. 


\section{Differential abundances at different taxonomic levels}

The 157 phylotype-OTUs (with $\geq 4$ members and prevalence in $>20 \%$ samples) were taxonomically placed (using RDP classifier and Greengene database) into a total of 91 genera with 88, 69, 88 and 87 genera in Aseel, broiler, Ghagus and Nicobari, respectively. At family level sequences were classified into a total of 68 families with 66, 54, 65 and 68 families in Aseel, broiler, Ghagus and Nicobari, respectively. At order level sequences were classified into a total of 44 orders with 42, 32, 42 and 44 orders in Aseel, broiler, Ghagus and Nicobari, respectively. At class level sequences were classified into a total of 35 classes with 34, 29, 35 and 35 classes in Aseel, broiler, Ghagus and Nicobari, respectively. At phylum level sequences were classified into a total of 20 phyla with 19, 18, 20 and 20 phyla in Aseel, broiler, Ghagus and Nicobari, respectively.

Out of 157 Phylotype-OTUs, edgeR analysis with FDR correction indicated that 88 phylotype-OTUs were significantly different in abundance between breeds/line. Major Phylotype-OTUs (top 41 out of 88 phylotype-OTUs in term of CSS normalized abundance) with significant difference in abundances between breeds/line along with taxonomy (last classified level) has been presented in Fig. 4. Many phylotype-OTUs containing large number of sequences had low taxonomic resolution (having a taxonomic resolution only down to the order level). Comparisons between group pairs using MannWhitney $U$ test indicated that among the 41 most abundant phylotype-OTUs there were significant difference in abundance of 24, 19, 21, 24, 26 and 6 phylotype-OTUs between Aseel vs broiler, Aseel vs Ghagus, Aseel vs Nicobari, broiler vs Ghagus, respectively. Interestingly, abundance of many phylotypeOTUs such as OTU1000062 (order Bacteroidales), OTU100296(family equivalent S24-7 uncultured gut microbial group), OTU102407 (genus Bacteroides), 0TU1057116 (phylum Bacteroidetes), OTU168571 (species Bacteroides barnesiae), OTU1758401 (genus equivalent SMB53 uncultured gut group) and OTU4324240 (genus Faecalibacterium) and were very low in the broiler line although these were highly abundant in all indigenous breeds. On the other hand abundance of OTU137026 (genus Lacobacillus), OTU1021172 (species Lactobacillus salivarius), OTU137026 (species Lactobacillus agilis), OTU181074 (genus equivalent CC115 gut group) and OTU549991 (species Lactobacillus helveticus) were higher in the broiler line than those of indigenous breeds.

Many phylotype OTUs were significantly higher in abundance in Aseel than in Ghagus or Nicobari. The notable ones, besides others, include OTU1000113(order Burkholderiales), OTU100567 (genus Ruminococcus), OTU839684 (family Lachnospiraceae), 0TU586453(family Christensenellaceae), OTU1010876(genus Oscillospira), OTU1057116(phylum Bacteroidetes) OTU 167741 (genus Dorea) and OTU1649772 (species Escherichia coli). On the other hand abundance of OTU1021172 (species Lactobacillus salivarius) and OTU1066621 (genus Prevotella) were higher in Ghagus than in Aseel. Abundance of few phylotype OTUs such as OTU100296 (family S24-7) and OTU1758401 (family SMB53) were lower in Nicobari than in Aseel or Ghagus. Abundance of OTU4369050 (family Fusobacteriaceae) was higher in Nicobari than in Aseel or Ghagus. Abundance of OTU1066621 (genus Prevotella) was significantly higher in Ghagus than in Aseel or Nicobari. 
Out of 91 genera having mean abundance of $\geq 4$ and prevalence of $>20 \%$, twenty four genera were significantly different in relative abundance between breeds/line. Genera having significant differences in abundance between breeds/line have been presented in Additional file 6 . Sequences not assigned to any genera remained major part of total sequences in all groups. Mann-Whitney $U$ test indicated that there were significant difference in abundance of $34,12,12,28,31$ and 4 genera between Aseel vs broiler, Aseel vs Ghagus, Aseel vs Nicobari, broiler vs Ghagus, broiler vs Nicobari and Ghagus vs Nicobari groups, respectively. The broiler line had significantly higher abundance of unclassified Bilophila, unclassified Blautia, unclassified cc_115, Defluvitella, Escherichia, Lactobacillus and unclassified Lactobacillus as compared to indigenous breeds. Indigenous breeds had significantly higher abundance of unclassified Bacteroides, Barnesiella, Faecalibacterium, Helicobacter, unclassified Odoribacter and unclassified Parabacteroides as compared to the broiler line. Some of the genera like Anaerorhabdus, Collinsella, Eubacterium, Marvinbryantia, unclassified Methanobrevibactor, unclassified Olsenella, p75_a5, unclassified Paraprevotella, Succinatimonas and Vestibaculum were not detected in broiler but were detected consistently, although in low numbers, in indigenous breeds. The genus Blautia was detected in all broiler samples and only in two samples of Aseel. The genus Eggerthella was only detected in the broiler line. The genus Anaerobiospirillum was detected in considerable numbers in Aseel and Nicobari but in only one sample of Ghagus. The genus Roseburia was detected only in Aseel and broiler but not in Ghagus or Nicobari.

Out of 72 families having mean abundance of $\geq 4$ and prevalence of $>20 \%$, metagenomeSeq analysis with FDR correction indicated that twenty eight families were significantly different in relative abundance between breeds/line. Families having significant difference in abundance between breeds/line have been presented in Additional file 7. Comparisons between different pairs of groups using Mann-Whitney $U$ test indicated significant difference in abundance of 21, 10, 12,20, 23 and 5 families between Aseel vs broiler, Aseel vs Ghagus, Aseel vs Nicobari, broiler vs Ghagus, broiler vs Nicobari and Gahgus vs Nicobari groups, respectively. The broiler line had significantly higher abundance of Lactobacillaceae as compared to indigenous breeds. Some of the prokaryaote families like unclassified Elusimicrobiaceae, Methanomassiliicoccaceae, Odoribacteriaceae, Oxalobacteraceae, Peptococcaceae, Peptostreptococcaceae, Porphyromonadaceae, Prevotellaceae, unclassified Rs_045, unclassified S24_7, unclassified Sphaerochaetaceae, unclassified Sphingobacteriaceae and Succinovibrionaceae were detected only in few samples in broiler but were detected more or less consistently although in low numbers in indigenous breeds. The family Ruminococcaceae as well as unclassified Ruminococcaceae was more abundant in Aseel and broiler followed by Ghagus. Large number of sequences remained unclassified upto family level. Abundance of Clostridiaceae was lowest in broiler followed by Nicobari.

Out of 45 orders meeting the minimum count and prevalence criteria, twenty two orders were significantly different in relative abundance between breeds/line. Orders having significant difference in abundance between breeds/line have been presented in Additional file 8. Comparisons between different pairs of groups using Mann-Whitney $U$ test indicated significant difference in abundance of 18,6, 12, 14, 17 and 1 orders between Aseel vs broiler, Aseel vs Ghagus, Aseel vs Nicobari, broiler vs Ghagus, broiler vs Nicobari and Gahgus vs Nicobari groups, respectively. The broiler line had significantly higher abundance of 
Enterobacteriales and Lactobacilales as compared to indigenous breeds. Indigenous breeds had significantly higher abundance of Bacteroidales and unclassified Bacteroidales as compared to the broiler line. Some of the orders like Anaeromonadales, Brachyspirales, CW040, Methanobacteriales, Sphaerochaetales, Spirochaetales and Turicbacterales were not detected in broiler but were detected consistently although in low numbers in indigenous breeds. The order Brachyspirales was only detected in Aseel and Nicobari. Abundance of Clostridiales was very high in Aseel and Broiler compared to Ghagus and Nicobari.

Class level abundance data of gut microbiota has been presented in Additional file 9. Out of 36 classes meeting the minimum count and prevalence criteria abundances of 30 classes were significantly different between groups. Comparisons between different pairs of groups using Mann-Whitney $\mathrm{U}$ test indicated significant difference in abundance of 24, 8, 14, 20, 20 and 9 classes between Aseel vs Broiler, Aseel vs Ghagus, Aseel vs Nicobari, Broiler vs Ghagus, Broiler vs Nicobari and Gahgus vs Nicobari groups, respectively. The broiler line had significantly higher abundance of Bacilli and Erysipelotrichi as compared to indigenous breeds. Indigenous breeds had significantly higher abundance of Bacteroidia as compared to the broiler line. Abundance of Clostridia was significantly higher in Aseel than Nicobari. Abundance of Clostridia was higher in broiler than those of Ghagus and Nicobari. Many classes were not detected consistently in broiler but were detected consistently although in low numbers in indigenous breeds.

Phylum level abundance data of gut microbiota has been presented in Additional file 10. Out of 19 Phyla edgeR analysis followed by FDR correction indicated that abundances of 5 phyla were significantly different between groups. Comparisons between different pairs of groups using Mann-Whitney $U$ test indicated significant difference in abundance of 3, 0, 5, 3, 3 and 1 phyla between Aseel vs broiler, Aseel vs Ghagus, Aseel vs Nicobari, broiler vs Ghagus, broiler vs Nicobari and Gahgus vs Nicobari groups, respectively. The broiler line had significantly higher abundance of Fimicutes as compared to indigenous breeds. Indigenous breeds had significantly higher abundance of unclassified Bacteroidetes as compared to the broiler line. Some of the phyla like Deferribacteres, Elusimicrobia, Spirochaetes, TM7 and unclassified WPS2 were either not detected or detected in few samples in broiler but were detected consistently although in low numbers in indigenous breeds. At phylum level there was significantly higher abundance of Actinobacteria, unclassified Bacteriodetes, Firmicutes and TM7 in Aseel as compared to Nicobari. There was no difference in abundance of any phylum between Aseel and Ghagus. Abundance of phylum TM7 was significantly higher in Ghagus than in Nicobari.

\section{The breed/Line specific biomarkers based on LEfSe algorithm}

The LEfSe analysis identified biomarkers in the gut microbiota (specific taxa that varied in abundance consistently by chicken breed or line) that were indicative of gut microbiota of each breed or line. In total, $82,35,54,37,27,21$ and 14 biomarkers were identified with LDA scores $>2.0$ at phylotype-OTU, species, genus, family, order, class and phylum levels, respectively. High abundance of genera Bacteroides, Oscillospira, Faecalibacterium, Coprococcus, Anaerobiospirillum, Sutterella, Olsenella, Paraprevotella, 
unclassified Clostridiacaea, Cloacibacillus, Turicibacter, Treponema, Collinsella, Succinatimonas, Gemmiger, Methanobrevibacter and Desulfovibrio were typical for Aseel breed (Fig. 5a). High abundance of genera or genus equivalent taxonomic groups Lactobacillus, Ruminococcus, unclassified Lactobacillaceae, Subdoligranulum, Dorea, cc_115, Blautia, Escherichia, Clostridium, Bilophila, Defluviitalea, Bifidobacterium and Eggerthella were typical for the broiler line. Similarly, higher abundance of few genera or genus equivalent taxonomic groups namely SMB53, Prevotella, Odoribacter, Parabacteroides, Akkermansia, YRC22 and RFN20 were typical to the Ghagus breed. Higher abundance of Fusobacterium, Meganomonas, Asteroleplasma, Barnesiella, Helicobacter, Elusimicrobia, WCHB1_41, Desulfovibrio, and Spirochaetes were typical to the Nicobari breed.

Cladogram of important biomarkers identified at different taxonomic levels in different breeds/line using LefSe with LDA scores $>3.5$ has been presented in Fig. 5 b. The class Coriobacteriia, orders such as unclassified Bacteroidetes and Aeromonadales, families like Christensenelaceae, Ruminococcaceae and Succinovibrionaceae were major biomarkers in the Aseel. The phylum Firmicutes, class Bacilli, order Enterobacteriales and families such as Lactobacillaceae and Enterobacteriacceae were top biomarkers in the broiler line. The phylum Bacteroidetes, classes TM7_3, Bacteroidia and Lentisphaeria, orders such as Bacteroidales and families like Odoribacteraceae, Paraprevotellaceae, Porphyromonadaceae, Prevotellaceae, S24_7, Clostridiaceae and Victivallaceae were top biomarkers in Ghagus. The phylum Elusimicrobia and Synergistetes, classes Fusobacteria, Elusimicrobia, Synergistia, Verruco_5, Epsilonproteobacteria, Betaproteobacteria and Pedosphaerae, orders such as Elusimicrobiales, Fusobacteriales, Burkholderiales and families such as Bacteroidaceae, Elusimicrobiaceae, Fusobacteriaceae, Alcaligenaceae and Synergistaceae were top biomarkers in Nicobari.

\section{The core gut microbiome}

Phylotype-OTUs with a mean normalized (CSS) relative abundance of at least $0.1 \%$ and having within breed prevalence of at least $50 \%$ in at least one breed were considered to define core microbiome in different breeds/line. Analysis of the prokaryotic community composition at the OTU level indicated that of the 296 phylotype-OTUs detected, only 22 phylotype-OTUs were present in $>0.1 \%$ relative abundance in at least $50 \%$ of the birds in at least one breed or line (Fig. 6). Twelve, five and two of the 22 phylotypeOTUs belonged to the phyla Firmicutes, Bacteroidetes and Proteobacteria, respectively with the rest belonging to other diverse phyla like Fusobacteria and Cyanobacteria. Only 5 phylotype-OTUs (OTU1000113 belonging to the order Clostridiales, OTU100567 belonging to the genus Ruminococcus, OTU1010876 belonging to the genus Oscillospira, OTU839684 belonging to the family Lachnospiraceae and OTU98948 belonging to the family Ruminococcaceae) occurred as core microbiome across all breeds or lines and these core OTUs represented $26 \%$ of total microbial count (Fig. 6). The OTU839684 belonging to the family Lachnospiraceae alone accounted for $13 \%$ of total microbiome (or $50 \%$ of core microbiome) count of all chicken breeds/line. Three phylotype-OTUs were unique to the broiler line (OTU181074 belonging to the genus level group cc_115, OTU2229500 belonging to the species Subdoligranulum variable, OTU549991 belonging to the species Lactobacillus helveticus). Two phylotype-OTUs were unique to the Aseel breed (OTU586453 assigned to the family Christensenellaceae 
and OTU60774 assigned to the species Anaerobiospirillum thomasii). One phylotype-OTU was unique to the Nicobari breed (OTU4369050 belonging to the genus Fusobacterium). Seven phylotype-OTUs (OTU1000062 belonging to the order Bacteroidales, OTU100296 belonging to the family level gut group S24_7, OTU102407 belonging to the genus Bacteroides, OTU1057116 belonging to the phylum Bacteroidetes, OTU1105376 belonging to the genus Suterella, OTU168571 belonging to the species Bacteroidea barnesiae and OTU4324240 belonging to the species Faecalibacterium prausnitzii) were core OTUs across the three indigenous breeds but were not consistently detected in the broiler line. These indigenous chicken specific core OTUs represented $49.3 \%$ of total prokaryote count of indigenous birds.

\section{Correlation analysis}

Family level correlations among microbes in Aseel, broiler, Ghagus and Nicobari are shown in Additional files 11, 12, 13 and 14, respectively. For Aseel, the occurrence of families which in- clude potentially pathogenic species such as Enterobacteriaceae, Clostridiaceae, Campylobacteraceae, Pasteurellaceae, Streptococcaceae, Staphylococcacae, Fusobacteriaceae, Enterococcaceae Corynebacteriaceae and Helicobacteraceae exhibited a positive correlation with each other, had a high positive correlation with Sutterellaceae, Oxalobacteraceae, Alcaligenaceae, Marinilabiacae, Victivallaceae, Deferribacteriaceae, Rhodospirillaceae, Methanocorpusculaceae, Flavobacteriaceae and Burkholderiaceae, and a high negative correlation with Christensenellaceae and Ruminococcacae and a low correlation with Lactobacillaceae and Bifidobacteriaceae. Families such as Lactobacillaceae, Peptococcaceae, Micrococcaceae, Actinomycetaceae, Bifidobacteriaceae, Lachnospiraceae, Coriobacteriaceae and Cytophagaceae exhibited a strong positive correlation with each other and a negative correlation with most other families. Surprisingly, Clostridiales family XIII Incertae sedis, Eubaccteriaceae, Methanobacteriaceae, Hyphomicrobiaceae and Moraxellaceae showed negative correlations with most of the families detected in Aseel (Additional file 11).

In case of the broiler line several small family level clusters with strong positive correlation with each other were detected. The families such as Sutarellaceae, Victivallaceae, Burkholderiaceae, Elusimicrobiaceae, Deferribacteriaceae, Rhodospirillaceae, Methanocorpusculacaea exhibited a strong positive correlation with each other. Campylobacteraceae, Fusobacteriaceae, Prevotellaceae, Rickenellaceae, Acidominococcaceae, Bacteroidaceae, and Desulfovibrionaceae exhibited a strong positive correlation with each other and negative correlation with most other families. Enterobacteriaceae, Pseudomonadaceae and Coriobacteriaceae showed a strong positive correlation with each other (Additional file 12).

In Ghagus several family level clusters of prokaryotes having strong positive correlations among themselves were detected such as :Suterellaceae, Victivallaceae, Burkholderiaceae, Elusimicrobiaceae, Deferribacteriaceae, Rhodospirillaceae, Methanocorpusculaceae, Sinobacteraceae, Erysipelotrichaceae and Alcaligenaceae, Lactobacillaceae, Desulfovibrionaceae and Enterobacteriaceae; Succinovibrionaceae, Helicobacteriaceae, Fusobacteriaceae, Bifidobacteriaceae and Peptostreptococcaceae, etc (Additional file 13). 
In Nicobari also several family level clusters which exhibited a strong positive correlation with each other were detected such as: Peptococcaceae, Lachnospiraceae, Coriobacteriaceae, Desulfovibrionaceae, Bifidobacteriaceae, Acidaminococcaceae, Lactobacillaceae, Christensenellaceae, Oxalobacteraceae, Prevotellaceae, Mycoplasmataceae, Enterobacteriaceae, Ruminococcaceae, Hyphomicrobiaceae, Erysipelotrichiaceae and Clostridiaceae, Fusobacteriaceae, Enterococcaceae, Veillonellaceae and Bacillaceae but had a negative correlation with Enterobacteriaceae, Coriobacteriaceae, Erysipelotrichaceae, Lachnospiraceae, Hyphomicrobiaceae and Lactobacillaceae. The families such as Methanocorpusculaceae, Victivallaceae, Fusobacteriaceae, Campylobacteriaceae, Prevotellaceae, Veillonellaceae, Verrucomicrobiaceae and Bacteroidaceae exhibited a strong positive correlation with each other with a negative correlation with Coriobacteriaceae, Enterobacteriaceae, Erysipelotrichaceae, Lachnospiraceae, Leuconodtocaceae, Clostridiaceae, Hyphomicrobiaceae and Lactobacillaceae. Similarly, Enterobacteriaceae, Erysipelotrichaceae and Coriobacteriaceae had a strong positive correlation with each other. Lactobacillaceae, Hyphomicrobiaceae and Clostridiaceae had a strong positive correlation with each other (Additional file 14).

\section{Correlation networks between microbiota}

The genus and order level correlation networks have been presented in Additional file 15 and Fig. 7, respectively. At genus level, significant pairwise correlations between different genera (with an absolute Spearman rank correlation coefficient of at least 0.5 and p-value cutoff of 0.05 ) resulted in a correlation network of 86 microbial genera (nodes) (out of 91) and 1061 edges (correlations > 0.5). At order level, significant pairwise correlations between different genera (with an absolute Spearman rank correlation coefficient of at least 0.5 and p-value cutoff of 0.05 ) resulted in a correlation network of 38 orders (nodes) (out of 44) and 247 edges (correlations > 0.5). At genus level most of the nodes had large number of edges or connections including many genera having low level of abundance. At order level the top hubs (nodes) of the network mainly consists of Burkholdales, Aeromonadales, unclassified Bacteroidales, Sphaerochaetales, Lactobacillales, E2, Elusimicrobiales, Synergistales, Bacteroidales and Fusobacteriales with $21,19,18,18,17,17,16,14,13$ and 12 connections, respectively.

\section{Discussion}

Demand for poultry meat and eggs are increasing rapidly in South Asia, where global commercial-type lines contribute most of the meat and egg production but popularity of indigenous chicken grown organically without antibiotic growth promoters are increasing at a relatively faster rate. Gut microbiota plays a key role in the development and functioning of gut including pathogen exclusion and therefore decreasing the expense of energy that birds normally invest in keeping the immune system active against these pathogens. It has been suggested that assessment of microbiome structure in gut in a comprehensive manner may further development of strategy for improving growth, feed efficiency, survivability or lower pathogen shedding via the development of host specific probiotics [4]. The advent of high throughput sequencing and omics approaches as tools for the study of microbial communities has allowed a detailed characterization of the gut microbiota of chickens in a quick and robust fashion, 
without the need to culture the microorganisms. This is the first study exploring gut microbiome of Nicobari and Ghagus breeds of Chicken breeds originated in Nicobari islands (a well known biodiversity hotspot) and coastal India, respectively. Here we have attempted to identify differences in gut microbial community structure of chicken breeds originated in diverse geographical locations and also with a global commercial broiler line.

The microbial communities differ through the chickens gut intestinal tract with particular microbial profiles detected in crop, gizzard, ileum, cecum and colon of broiler chickens [23]. Here we have analysed microbiota from entire hindgut (duodenum to cloaca including caecum) to focus on segments generally considered to be most important for gut health and function.

The initial colonization of the gastrointestinal tract of birds occurs naturally after hatching and can even begin before, by passing of microorganisms through the pores of the eggshell [24]. After the initial colonization of the intestine the species richness and complexity of the population structure of the microbiota increase as the birds grow, until microbiota reaches a state of stabilization. This process normally occurs in commercial broiler chickens around 3 weeks of life [25]. However, development times and succession patterns of intestinal microbiota species can vary depending on the genetic makeup of the birds and management factors. Here, we selected time points late in the production cycle of each breed or line (as each breed matures i.e., at marketing age) to permit assessment of the outcome of colonisation throughout each chickens' life.

In the present study indigenous chicken breed presented higher number of OTUs (1767 to 3461 ) than that of the commercial broiler line (857). In contrast, in an earlier study the commercial broiler line Cobb 400 was reported to have more OTUs (1273) than Indian indigenous chicken breeds such as Aseel (735 to 1134) or Kadaknath (816 to 833). In general, more number of OTUs were detected in this study as compared to that of Pandit et al.[6]. This may be partly attributed to the fact that in the current study no antibiotic growth promoter (AGP) was used and we have analysed gut content from entire hindgut (duodenum to cloaca including caeca) whereas in the study reported by Pandit et al. [6] only cecal content of birds fed diet containing AGP were analysed.

A total of 135 (out of 296) phylotype-OTUs (with > 2 members) were shared by chickens from all groups.

The gut microbiota of Indian native chicken breeds evaluated here were dominated by sequences belonging to the phyla Bacteroidetes and Firmicutes whereas the gut microbiota of the commercial line was dominated by sequences representative of the phylum Firmicutes. Within Indian indigenous breeds, Firmicutes/Bacteroidetes ratio were more and less similar in Ghagus and Nicobari breed but substantially different from that of Aseel. Our data are in discordance with a previous report by Saxena et al. [5] showing the dominance of Firmicutes in gut microbiome of Asamese breed of Indian Indigenous chicken. Pandit et al. [6] reported that Bacteroidetes was the dominant phyla in most of the gut caecal samples of Indian indigenous breeds like Kadaknath and Aseel and Firmicutes were more common in Cobb 400 samples, which is in concordance with our current study. Generally higher Firmicutes / Bacteroidetes ratios have been shown to be correlated with obesity in human [26]. Both Firmicutes and Bacteroidetes 
are primarily carbohydrate fermenters. Firmicutes are known to produce both butyrate and propionate, whereas Bacteroidetes primarily produce propionates as fermentation end product [27]. Within Firmicutes, different genera under the order Clostridiales were predominant in different breeds or line. The Bacilli members, like Lactobacillus spp., possessing prebiotic and probiotic activities, were present in very high proportions in the broiler line but were in small proportions in indigenous breeds. However, the genus Faecalibacterium (under order Clostridiales and family Ruminococcaceae), known to produce butyrate and thus having a crucial role in maintaining gut health and host well being [28], were present in significant proportion in indigenous breeds but not in the broiler line. The genus Bacteroides and an unknown genus under the order Bacteroidales constituted the top two dominant genera in all the indigenous breeds, whereas Lactobacillus and an unknown genus under the family Lachnospiraceae constituted the top two dominant genera in the boiler line. It has been shown that Faecalibacterium cooccurs with several members of Bacteroidetes in gut [29] and it has been suggested that Faecalibacterium may rely on Bacteroides for cross feeding [28]. Interestingly, as in case of broiler, an unknown genus under the family Lachnospiraceae constituted very high proportion (11\%) of microbiota in Aseel but were detected in relatively lower proportion in other indigenous breeds. Gut microbial composition is mediated by many factors such as geographical location, host diet and administration of antibiotics and other medicines. It has been shown that the succession of changes in gut microbiota correlates with changes in the cytokine profile expressed by host intestinal cells [30].

In the present study alpha diversity estimators measuring species richness (Chao1and ACE) as well as diversity (Simpson, Shannon and Fisher) and rarefaction curve indicated that the broiler line VenCobb 400 had lower diversity than those of the indigenous breeds, in disagreement with a previous study [6].

Beta diversity analyses were carried out using different types of distance or dissimilarity metrices. The Jaccard distance is based on presence or absence of OTU/species and does not include abundance information. The Bray - Curtis dissimilarity measure is based on abundance of each OTU/species in different communities and it ignore cases in which the species /OTU is absent in both community and is dominated by the abundant species so that rare species add very little to the value of the coefficient [31]. The Jensen-Shannon distance is based on probability distribution of two microbial communities [32] and is a measure of divergence between distributions accounting for both presence and abundances of OTUs. UniFrac is a distance measure based on phylogenetic information (degree of phylogenetic divergence or distance) of OTUs of microbial communities. Unweighted UniFrac measures the distance between two communities by calculating the fraction of the branch length in a phylogenetic tree that leads to descendants in either, but not both, of the two communities and is considered as a qualitative measure of diversity which takes into account presence/absence of data but does not include abundance data to compare community composition. Weighted UniFrac is a variant of the original unweighted UniFrac measure that weights the branches of a phylogenetic tree based on the abundance of information and is considered as a quantitative measure of diversity, which also take the relative abundance of each type of organism into account [33]. 
Beta diversity analysis involving different distance metrices has indicated clear separation of microbiome at both OTU as well as phylum levels by chicken breed or line indicating a strong host component in microbiome composition, in agreement with previous studies [6, 34, 35]. Low levels of overlap between microbiome of the broiler line with those of indigenous breeds also suggest highly different microbial composition across these two categories. Interestingly, NMDS plots also indicates that compositional distribution of gut microbiome of the island breed (Nicobari) have very high degree of similarity with both the coastal breed (Ghagus) and the breed from Indian mainland (Aseel) indicating possibility of strong evolutionary linkage among all these indigenous breeds.

Slightly different results between unweighted unifrac and weighted unifrac beta diversity metrices suggest that the most abundant taxa were more phylogenetically related compared to the low abundant taxa. Weighted unifrac distance considers presence or absence, abundance and phylogenetic relatedness and is considered as a measure of most recent common ancestor and also gives more weightage to deep branches of phylogenetic tree, whereas unweighted unifrac distance only consider presence and absence and no consideration of abundance and gives less importance to deep branches and is influenced by all of the branches [36].

Differential abundance analysis using edgeR indicated that abundances of several phylotype-OTUs belonging to the order Bacteroidales and an phylotype-OTU under genus Faecalibacterium were significantly lower in the broiler line VenCobb 400 but abundances of few phylotype-OTUs belonging to the genera such as Lactobacillus, Bilophila, Clostridium, Weissella, Eggerthella and Defluvitalea were higher $(P<0.05)$ in the broiler line as compared to indigenous breeds.

At order level 18,6,12,14, 17 and 1 orders were differentially abundant between Aseel vs broiler, Aseel vs Ghagus, Aseel vs Nicobari, broiler vs Ghagus, broiler vs Nicobari and Ghagus vs Nicobari, respectively. Similar trend was also observed at other taxonomic levels indicating highest overlap in microbial community composition between the coastal breed (Ghagus) and the island breed (Nicobari) among indigenous breeds and very low overlap between the broiler line and all indigenous breeds.

LEfSe biomarkers also indicated a possible strong host genetic influence on gut microbiome.

Breed or line specific potential pathogenic and/or zoonotic organisms were also detected as biomarkers such as : Bilophila, Escherichia, Clostridium and Eggerthella in broiler; Anaerobiospirillum, Sutterella, Turicibacter, Collinsella and Treponema in Aseel; SMB53 gut microbial group in Ghagus; Fusobacterium, Asteroplasma, Helicobacter and Elusimicrobia in Nicobari.

On the other hand many breed or line specific prokaryotes having potential beneficial effects on gut health of hosts (having probiotic or butyric acid producing properties) were also associated as biomarkers such as: Faecalibacterium, Coprococcus, Gemmiger in Aseel; Lactobacillus, Subdoligranulum, Dorea, Blautia and Bifidobacterium in the broiler line; Akkermansia in Ghagus; Barnesiella in Nicobari. 
Using $0.1 \%$ relative abundance and $50 \%$ prevalence cutoff criteria, 22 phylotype OTUs out of 296 phylotype OTUs representing $26 \%$ of total microbial count were found to qualify as core OTUs in at least one breed or line. In contrast, only 5 phylotype-OTUs representing $13 \%$ of total microbiome occurred as core microbiome across all breeds or line.

Interestingly, few potentially pathogenic or harmful ( Sutterella in Aseel and Nicobari, Fusobacterium in Nicobari and Anaerobiospirillum in Aseel) prokaryotes were detected above the abundance and prevalence cutoffs thus qualifying to be part of core microbiome in different breeds or line. This is in line with an earlier report where Campylobacter were detected above the 1.0\% cut-off in Kadaknath and Aseel, but not in Cobb400 or Ross 308 [6] .

In contrast many prokaryotes having potential beneficial effects on gut health of hosts (having probiotic or butyric acid producing properties) were also detected above the $0.1 \%$ relative abundance and $50 \%$ prevalence cutoff in different breeds and lines.

Futher work will be required to ascertain exact role of the pathogenic bacteria detected as biomarkers or core microbiome. However, besides being opportunistic or primary pathogens, inflammatory and immune responses induced by these pathogens have been suggested to influence the intestinal environment, host immunity and its bacterial communities [37, 38].

Despite inter-individual differences in community composition, a core set of microbes shared across individuals of a particular breed suggesting presence of breedwise distinct community composition. Millions of years of co-evolution between the host and microbes have led to a mutualistic symbiosis in which the microbiota contributes to many host physiological processes and the host, in turn, provides a nutritious and hospitable environment to the microbes. Further, the normal gut symbionts forms a stable community that resists the invasion and colonization of non-native bacteria [39].

Potentially opportunistic pathogens such as those within the families Enterobacteriaceae, Clostridiaceae, Campylobacteriaceae, Fusobacteriaceae. etc exhibited a strong correlation with each other and a negative correlation with beneficial bacteria, having probiotic (competitive exclusion) properties or SCFA production ability, belonging to families such as Christensenellaceae, Lactobacillaceae and Bifidobacteriaceae. Several breed or line specific microbial family level clusters with strong positive correlations with each others and negative correlations with other clusters were detected in all the breeds or lines suggesting existence of strong interactions among different microbial groups. Correlation network analysis data both at genus and order levels further substantiates existence of diverse and complex microbial networks. This is in line with earlier reports $[6,40]$.

\section{Conclusions}

In this study, we carried out the first comprehensive analysis of chicken gut microbiome of Nicobari and Ghagus breed of Indian native chicken originated in biodiversity hotspots like Nicobari island and coastal India, respectively and also carried out comparative analysis of diversity and composition of gut 
microbiota of three indigenous breeds and one commercial broiler line. The study presented here provides important insights into chicken breed or line specific variation in enteric bacterial occurrence, diversity and complex microbial networks. Our amplicon sequencing results emphasized the more similarity of the microbiota within the gut lumen of indigenous breeds as compared to the commercial broiler line VenCobb 400, but exhibited distinctive taxonomic differences between them as well. The study also indicated that among native breeds there is more similarity of gut microbiome of the island breed (Nicobari) with that of the coastal breed (ghagus) as compared to the mainland breed (Aseel). The study presented here indicated existence of breed or line specific core microbiome as well as across -breed or line core microbiome in chicken and occurrence of both beneficial and potential opportunistic pathogenic microbes as part of core microbiome. A deeper understanding of host-microbiome interactions as emanated from the current study may support development of strategies including development of breed specific feed additives and probiotics for enhanced productivity from unconventional or low value diets, for prevention of colonisation by pathogenic and zoonotic organisms and for deleopment of alternative to antibiotic growth promoters.

\section{Declarations}

\section{Acknowledgements}

Not applicable.

\section{Funding}

This research work is funded by ICAR Directorate of Poultry Research.

\section{Availability of data and materials}

The sequences for this study has been deposited in the Sequence Read Archive of the NCBI (accession numbers: PRJNA641245 and PRJNA641779).

\section{Authors' contributions}

SSP did data analysis and manuscript writing; RNC and SSP designed the study and analysis plan. BP, SPY and AK did preliminary data processing and literature review and RNC,SVR and MVL did manuscript correction

\section{Ethics approval}

This study was carried out using welfare standards consistent with those established under Indian Law. All protocols were approved by the Institute Animal ethics committee of ICAR Directorate of Poultry Research

\section{Consent for publication}


Not applicable.

\section{Competing interests}

The authors declare that they have no competing interests.

\section{References}

1. Thomas M, Wongkuna S, Ghimire S, Kumar R, Antony L, Doerner KC, et al. Gut Microbial Dynamics during Conventionalization of Germfree Chicken. mSphere. 2019;4:e00035-19. doi:10.1128/mSphere.00035-19.

2. Sommer F, Bäckhed F. The gut microbiota-masters of host development and physiology. Nat Rev Microbiol. 2013;11:227-38.

3. Wallace RJ, Sasson G, Garnsworthy PC, Tapio I, Gregson E, Bani P, et al. A heritable subset of the core rumien microbiome dictates dairy cow productivity and emissions. Sci Adv. 2019;5:eaav8391. doi:10.1126/sciadv.aav8391.

4. Singh KM, Shah T, Deshpande S, Jakhesara SJ, Koringa PG, Rank DN, et al. High through put $16 \mathrm{~S}$ rRNA gene-based pyrosequencing analysis of the fecal microbiota of high FCR and low FCR broiler growers. Mol Biol Rep. 2012;39:10595-602.

5. Saxena S, Saxena V, Tomar S, Sapcota D, Gonmei G. Characterization of caecum and crop microbiota of Indian indigenous chicken targeting multiple hypervariable regions within 16 s rRNA gene. Br Poult Sci. 2016;57:381-9.

6. Pandit RJ, Hinsu AT, Patel NV, Koringa PG, Jakhesara SJ, Thakkar JR, et al. Microbial diversity and community composition of caecal microbiota in commercial and indigenous Indian chickens determined using 16 s rDNA amplicon sequencing. Microbiome. 2018; 6: 115;doi;10.1186/s40168018-0501-9.

7. Chatterjee RN, Rai RB, Pramanik SC, Sunder J, Senani S, Kundu A. Comparative growth, production, egg and carcass traits of different crosses of Brown Nicobari with White Leghorn under intensive and extensive management systems in Andaman, India. Livest Res Rural Dev. 2007;19:193.

8. Rajkumar U, Haunshi S, Paswan C, Raju MVLN, RamaRao SV, Chatterjee RN. Characterization of indigenous Aseel chicken breed for morphological, growth, production and meat composition traits from India. Poult Sci. 2017;96:2120-6.

9. Yadav SP, Kannaki TR, Mahapatra RK, Paswan C, Bhattacharya TK, Sarkar SK, et al. In vivo cellmediated immune, hemagglutination inhibition response, hematological and biochemical values in native vs. exotic chicken breeds. Poult Sci. 2018;97:3063-71.

10. Schloss PD. Reintroducing mothur: 10 Years Later. Appl Environ Microbiol. 2020;86:e02343-19. doi:10.1128/AEM.02343-19.

11. Klindworth A, Pruesse E, Schweer T, Peplies J, Quast C, Horn M, et al. Evaluation of general 16S ribosomal RNA gene PCR primers for classical and next-generation sequencing-based diversity 
studies. Nucleic Acids Res. 2012. doi:10.1093/nar/gks808.

12. Rognes T, Flouri T, Nichols B, Quince C, Mahé F. VSEARCH: a versatile open source tool for metagenomics. PeerJ. 2016;4:e2584. doi:10.7717/peerj.2584.

13. Wang Q, Garrity GM, Tiedje JM, Cole JR. Naive Bayesian Classifier for Rapid Assignment of rRNA Sequences into the New Bacterial Taxonomy. Appl Environ Microbiol. 2007;73:5261-7.

14. Wei ZG, Zhang SW. A Dynamic Multi-Seeds Method for Clustering 16S rRNA Sequences Into OTUs. Front Microbiol. 2019;10:428. doi:10.3389/fmicb.2019.00428.

15. Wang Y, Xu L, Gu YQ, Coleman-Derr D. MetaCoMET: a web platform for discovery and visualization of the core microbiome. Bioinformatics. 2016;32:3469-70.

16. Arndt D, Xia J, Liu Y, Zhou Y, Guo A, Cruz J, et al. METAGENassist: a comprehensive web server for comparative metagenomics. Nucl Acids Res. 2012;40:W88-95.

17. Dhariwal A, Chong J, Habib S, King IL, Agellon LB, Xia J. MicrobiomeAnalyst: a web-based tool for comprehensive statistical, visual and meta-analysis of microbiome data. Nucleic Acids Res. 2017;3(W1):W180-8. doi:10.1093/nar/gkx295. 45 ) .

18. SPSS Inc. Released 2008. SPSS Statistics for Windows, Version 17.0. Chicago: SPSS Inc.

19. Segata N, Izard J, Waldron L. Gevers D, Miropolsky L, Garrett WS, et al. Metagenomic biomarker discovery and explanation. Genome Biol. 2011; 12: R60; doi:10.1186/gb-2011-12-6-r60.

20. Ondov BD, Bergman NH, Phillippy AM. Interactive metagenomic visualization in a Web browser. BMC Bioinformatics. 2011;12:385.

21. Heck KL, Belle GV, Simberloff D. Explicit calculation of the rarefaction diversity measurement and the determination of sufficient sample size. Ecology. 1975;56:1459-61.

22. McCall L, Callewaert C, Zhu Q, Song S, Bouslimani A, Minich JJ, et al. Home chemical and microbial transitions across urbanization. Nat Microbiol. 2020;5:108-15.

23. Sekelja M, Rud I, Knutsen SH, Denstadli V, Westereng B, Næs T, et al. Abrupt Temporal Fluctuations in the Chicken Fecal Microbiota Are Explained by Its Gastrointestinal Origin. Appl Environ Microbiol. 2012;78:2941-8.

24. Lee S, La T-M, Lee H-J, Choi I-S, Song C-S, Park S-Y, Lee J-B, Lee S-W. Characterization of microbial communities in the chicken oviduct and the origin of chicken embryo gut microbiota. Sci Rep. 2019;9:6838. doi:10.1038/s41598-019-43280-w.

25. Diaz Carrasco JM, Casanova NA, Fernández Miyakawa ME, Microbiota. Gut Health and Chicken Productivity: What Is the Connection? Microorganisms. 2019;7:374. doi:10.3390/microorganisms7100374.

26. Turnbaugh PJ, Hamady M, Yatsunenko T, Cantarel BL, Duncan A, Ley RE, et al. A core gut microbiome in obese and lean twins. Nature. 2009;457:480-4.

27. Aguirre M, Eck A, Koenen ME, Svalkoul PHM, Budding AE, Venema K. Diet drives quick changes in the metabolic activity and composition of human gut microbiota in a validated in vitro gut model. Res Microbiol. 2016;167:114-25. 
28. Lopez-Siles M, Duncan S, Garcia-Gil L, Mrtinez-Medina M. Faecalibacterium prausnitzii: from microbiology to diagnostics and prognostics. ISME J. 2017;11:841-52.

29. Qin J, Li R, Raes J, Arumugam M, Burgdorf KS, Manichanh C, et al. A human gut microbial gene catalogue established by metagenomic sequencing. Nature. 2010;464:59-65.

30. Oakley BB, Kogut MH. Spatial and Temporal Changes in the Broiler Chicken Cecal and Fecal Microbiomes and Correlations of Bacterial Taxa with Cytokine Gene Expression. Front Vet Sci. 2016;3:11.

31. Bray JR, Curtis JT. An ordination of upland forest communities of southern Wisconsin. Ecol Monogr. 1957;27:325-49.

32. Endres DM, Chindelin JE. A new metric for probability distribution. IEEE Trans Inf Theory. 2003;49:1858-60.

33. Catherine A, Lozupone MH, Kelley ST, Knight R. Quantitative and Qualitative $\beta$ Diversity Measures Lead to Different Insights into Factors That Structure Microbial Communities. Appl Environ Microbiol. 2007;73:1576-85.

34. Salanitro J, Fairchilds I, Zgornicki Y. Isolation, culture characteristics, and identification of anaerobic bacteria from the chicken cecum. Appl Microbiol. 1974;27:678-87.

35. Zhao L, Wang G, Siegel P, He C, Wang H, Zhao W, et al. Quantitative genetic background of the host influences gut microbiomes in chickens. Sci Rep. 2013;3:1163.

36. Fukuyama J. Emphasis on the deep or shallow parts of the tree provides a new characterization of phylogenetic distances. Genome Biol. 2019;20:131. doi:10.1186/s13059-019-1735-y.

37. Humphrey S, Chaloner G, Kemmett K, Davidson N, Williams N, Kipar A, et al. Campylobacter jejuni is not merely a commensal in commercial broiler chickens and affects bird welfare. MBio. 2014;5:e01364-14.

38. Jung A, Rautenschlein S. Comprehensive report of an enterococcus cecorum infection in a broiler flock in Northern Germany. BMC Vet Res. 2014;10:311.

39. Pickard JM, Zeng MY, Caruso R, Núñez G. Gut microbiota: Role in pathogen colonization, immune responses, and inflammatory disease. Immunol Rev. 2017;279:70-89. doi:10.1111/imr.12567.

40. Huang $P$, Zhang $Y$, Xiao K, Jiang F, Wang $H$, Tang D, et al. The chicken gut metagenome and the modulatory effects of plant-derived benzylisoquinoline alkaloids. Microbiome. 2018;6:21. doi:10.1186/s40168-018-0590-5.

\section{Figures}



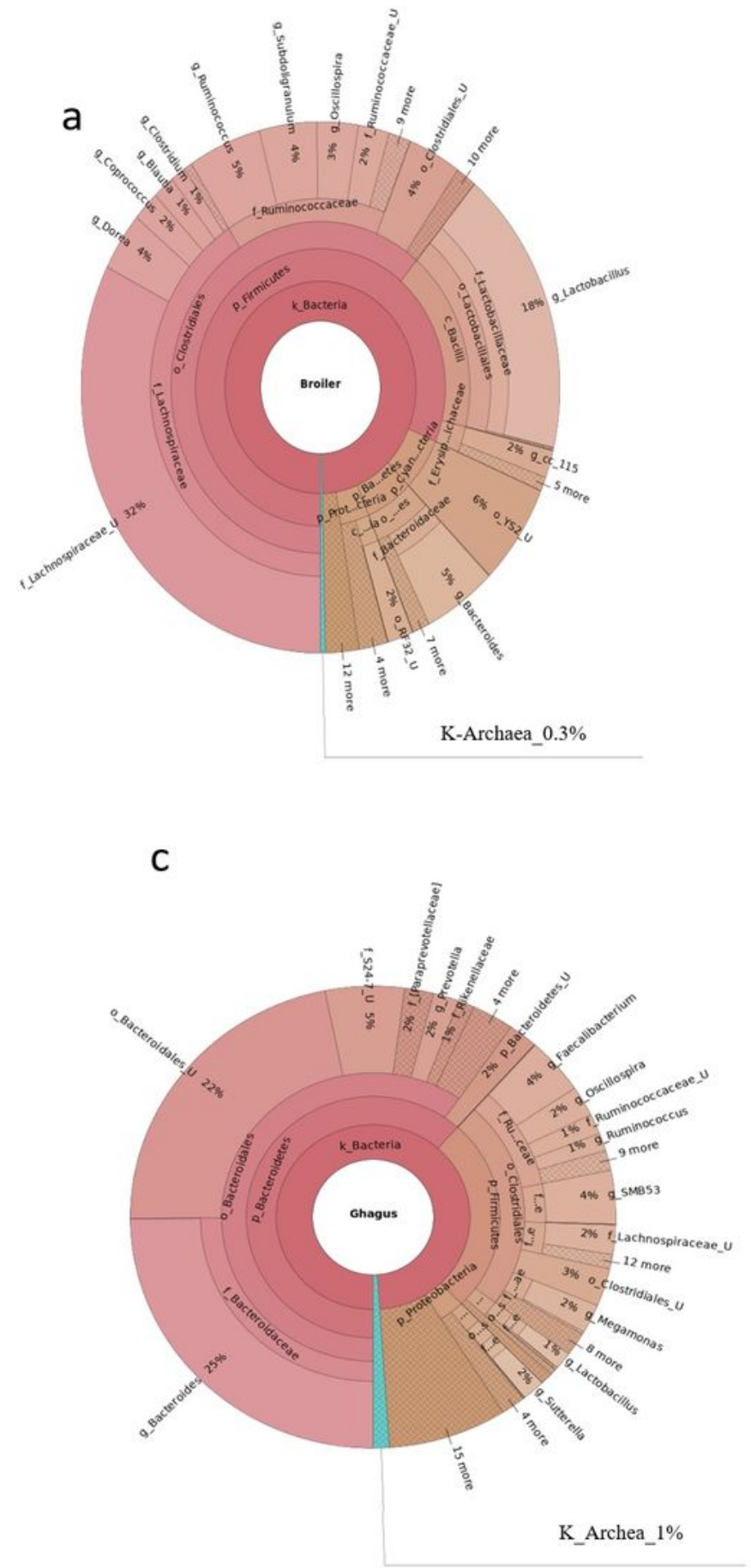

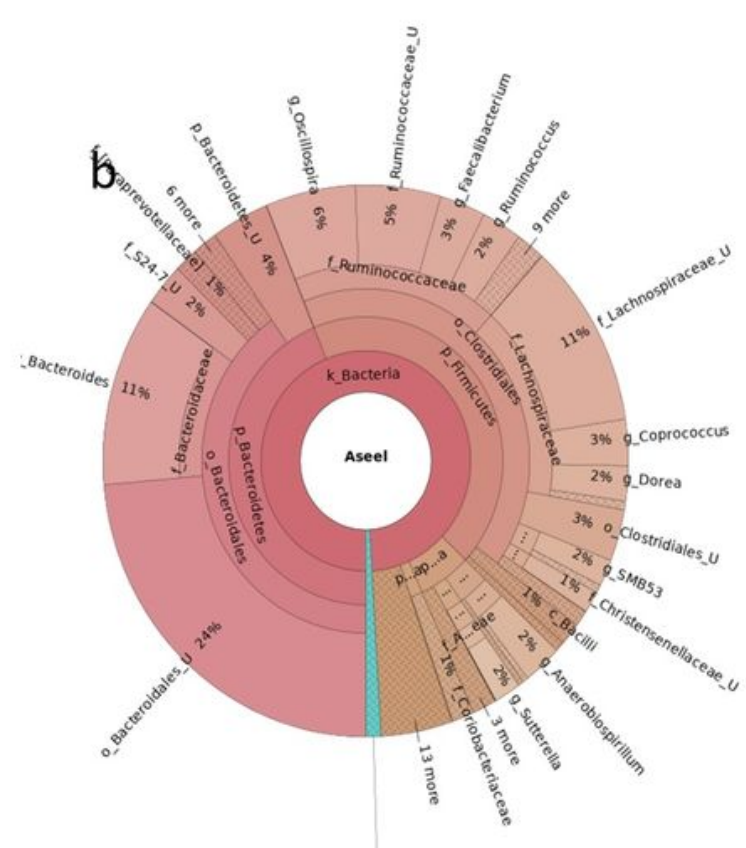

K_Archaea_0.9\%

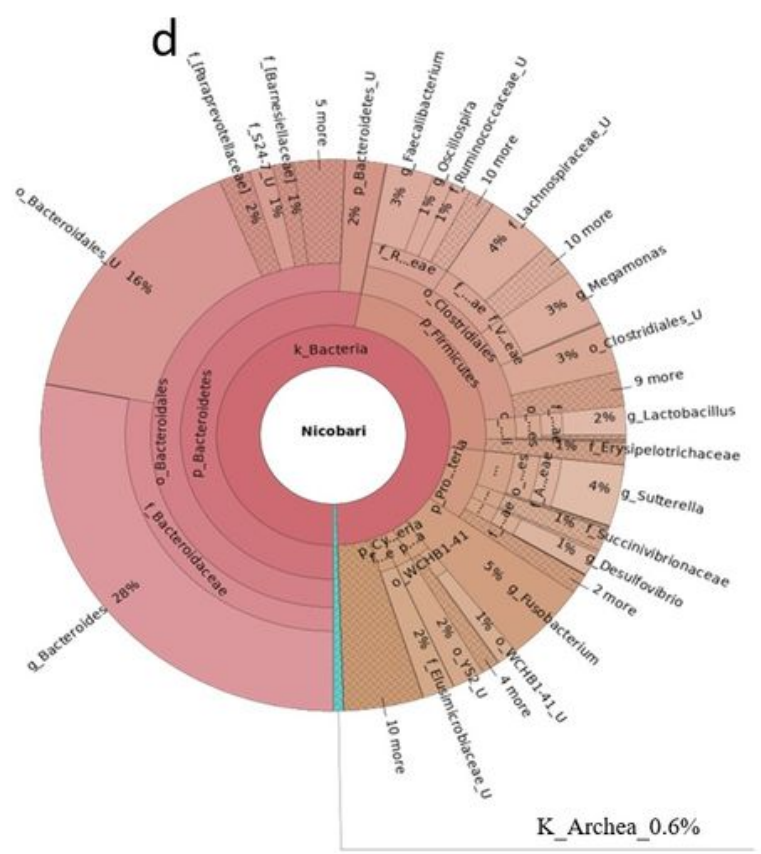

\section{Figure 1}

Krona chart of taxonomic assignments. Mean relative abundance of the prokaryotes at different taxonomic levels in different breeds or line are shown as Krona charts for (a) broiler line VenCobb 400, (b) Aseel, (c) Ghagus, (d) Nicobari. 

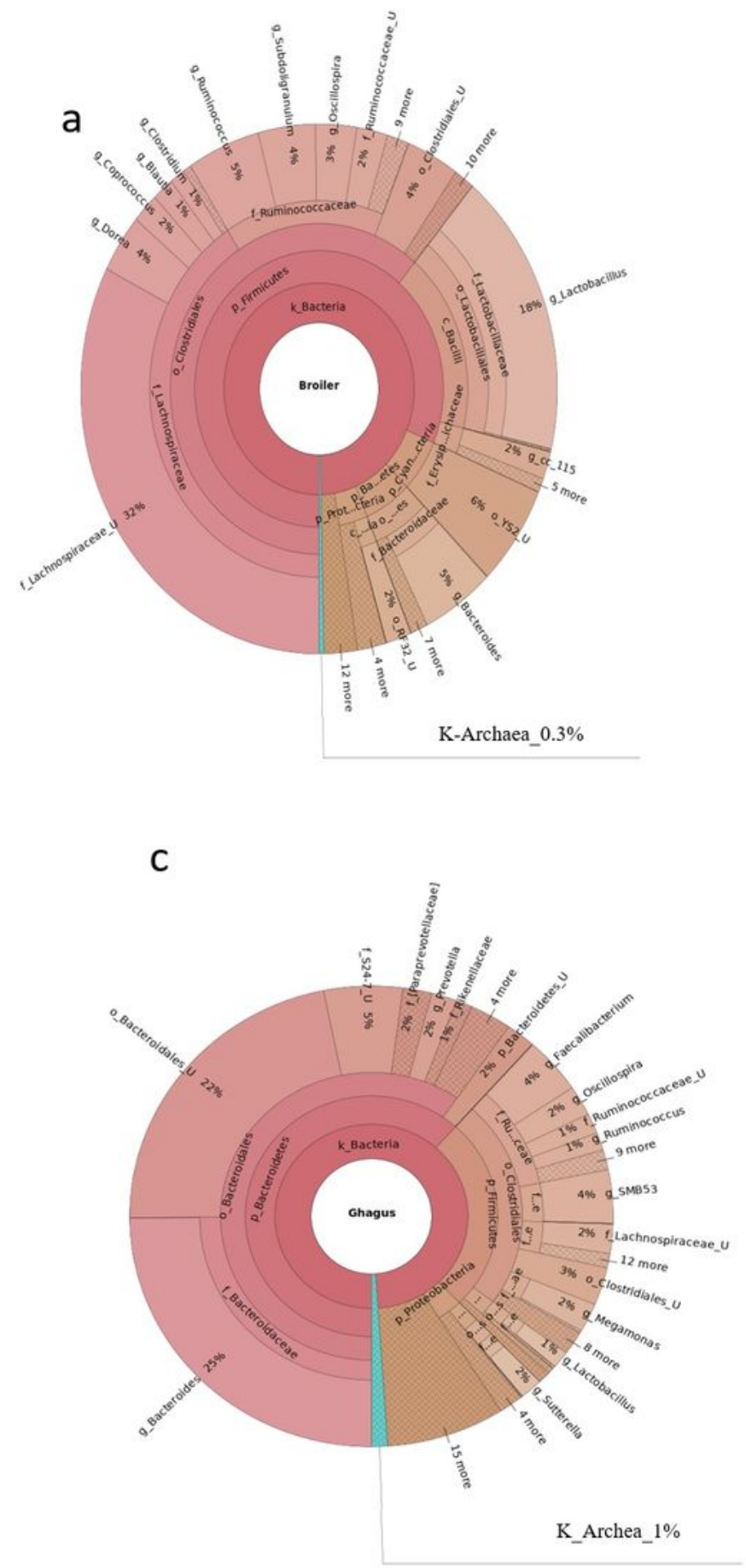

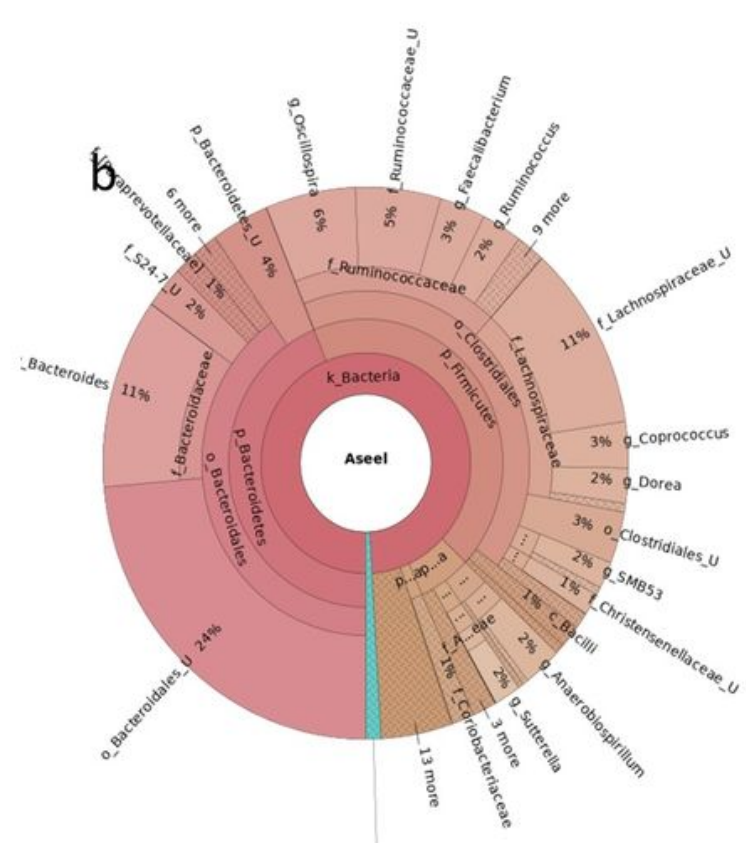

K_Archaea_0.9\%

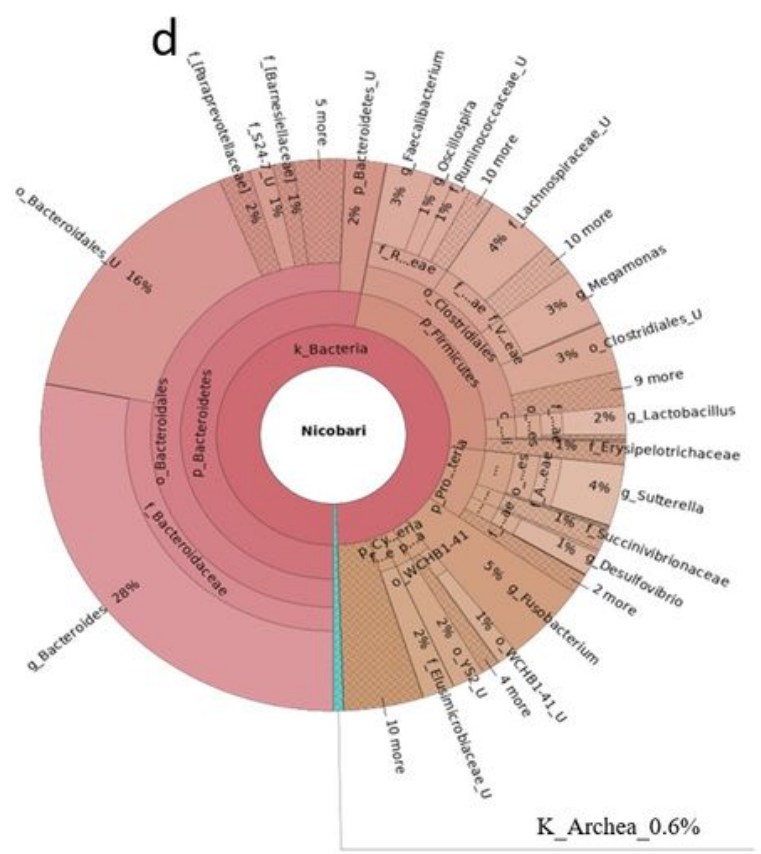

\section{Figure 1}

Krona chart of taxonomic assignments. Mean relative abundance of the prokaryotes at different taxonomic levels in different breeds or line are shown as Krona charts for (a) broiler line VenCobb 400, (b) Aseel, (c) Ghagus, (d) Nicobari. 

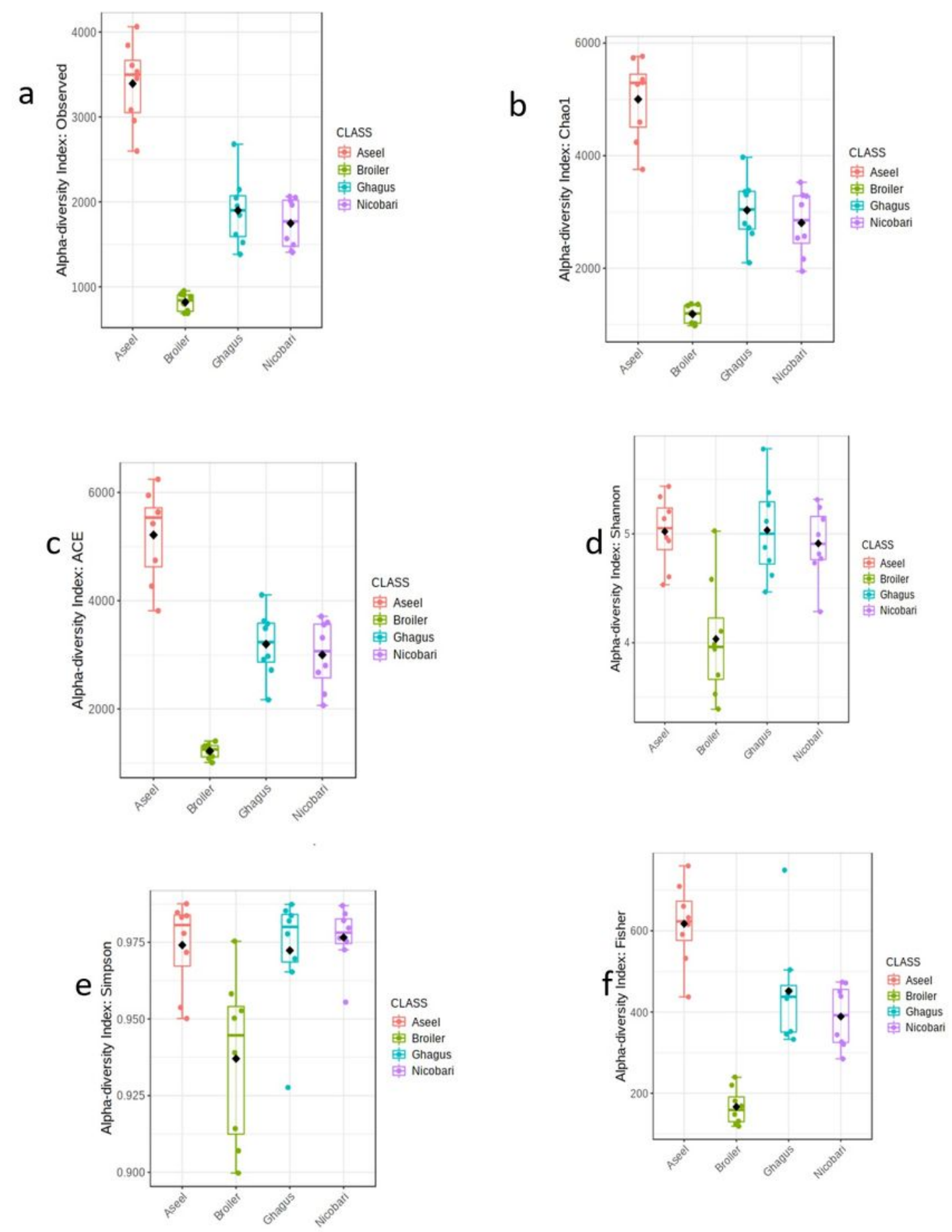

\section{Figure 2}

Alpha diversity across samples. Differences in alpha diversity metrices of microbial diversity and richness in gut microbiota of chickens of different breeds or line are shown as boxplots. (a) observed number of OTUs, (b) Chao1, (c) ACE, (d) Shannon, (e), Simpson, (f) Fisher 

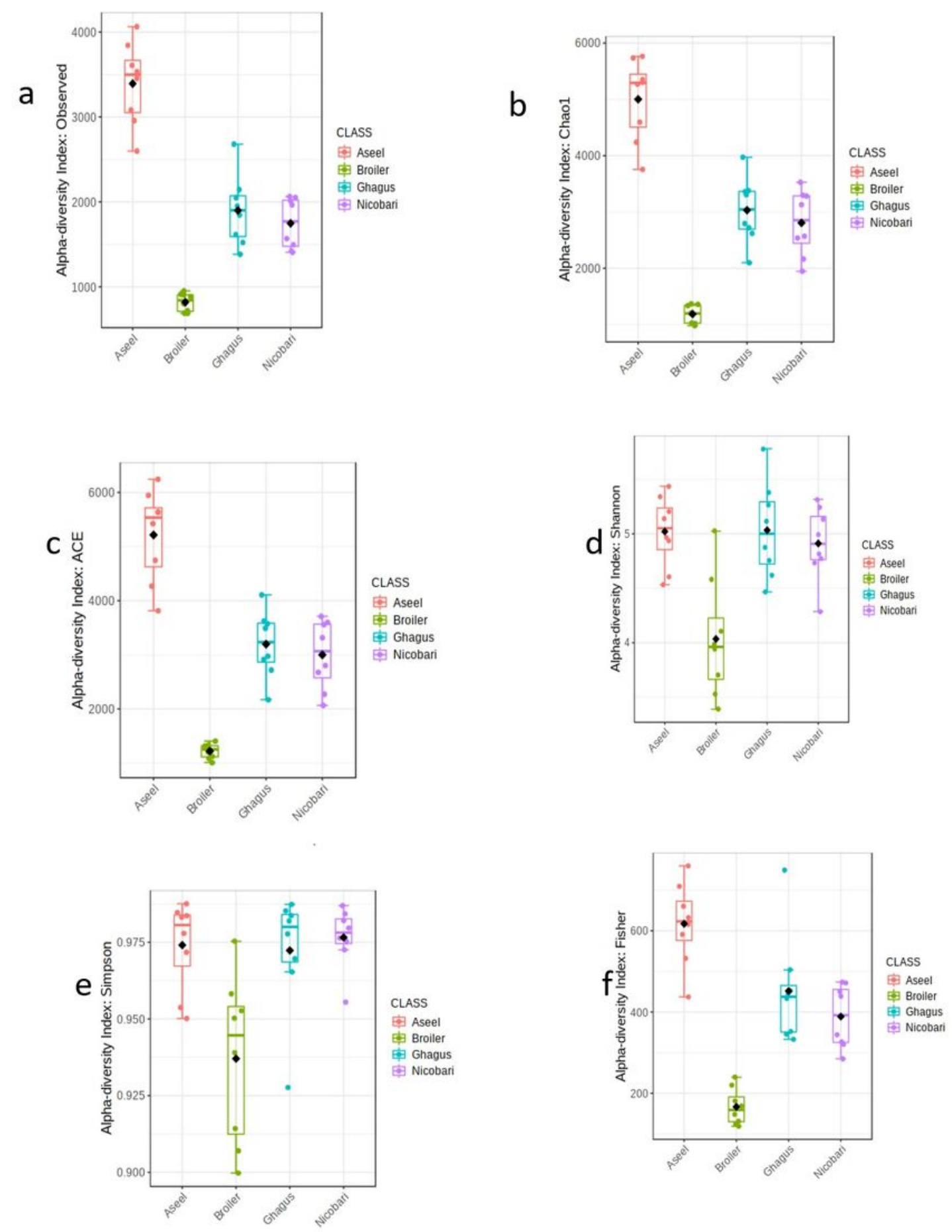

\section{Figure 2}

Alpha diversity across samples. Differences in alpha diversity metrices of microbial diversity and richness in gut microbiota of chickens of different breeds or line are shown as boxplots. (a) observed number of OTUs, (b) Chao1, (c) ACE, (d) Shannon, (e), Simpson, (f) Fisher 


\section{NMDS_Jensen-Shannon_OTU; \\ Stress $=0.091409$}

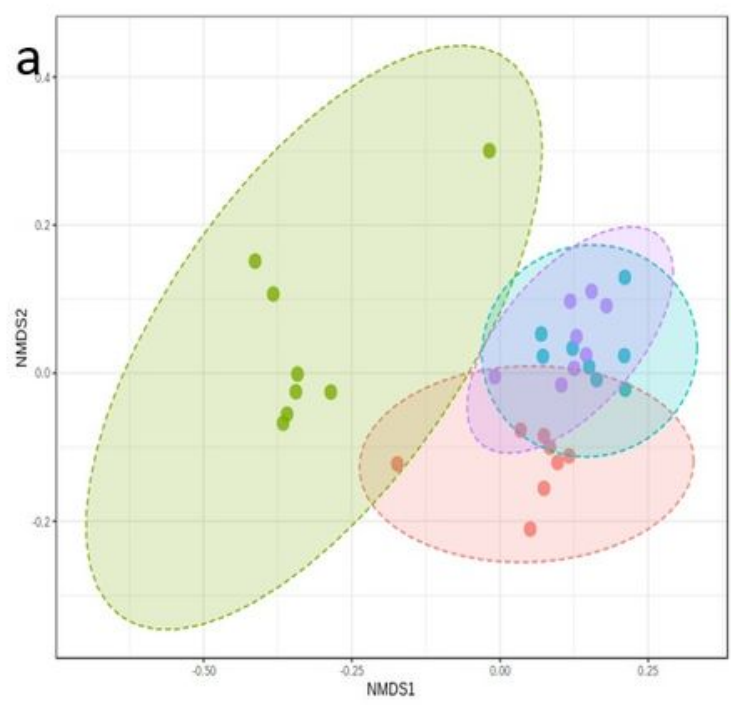

NMDS_unweighted UniFrac_ OTU; Stress $=0.069008$

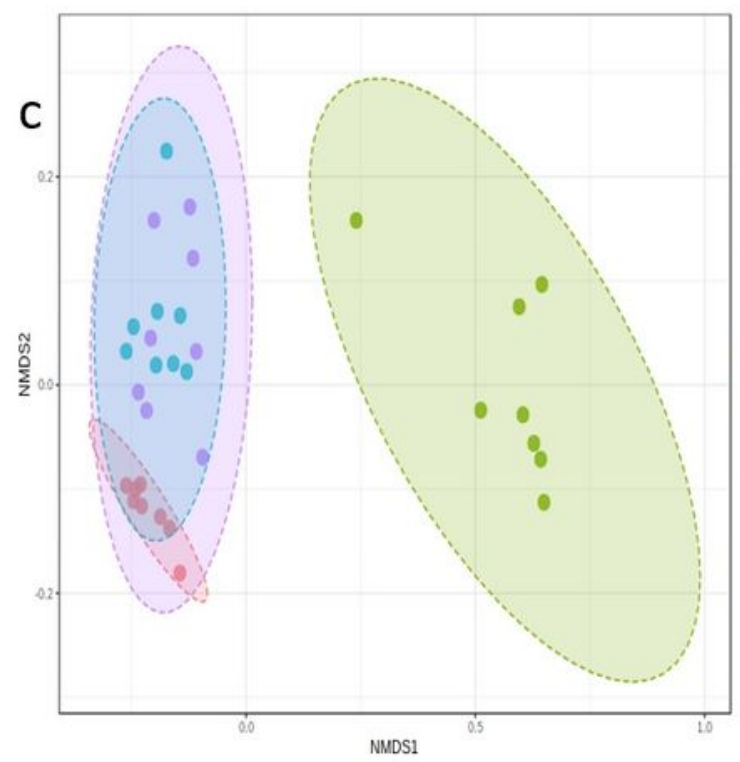

NMDS_Weighted UniFrac OTU;

Stress $=0.062276$

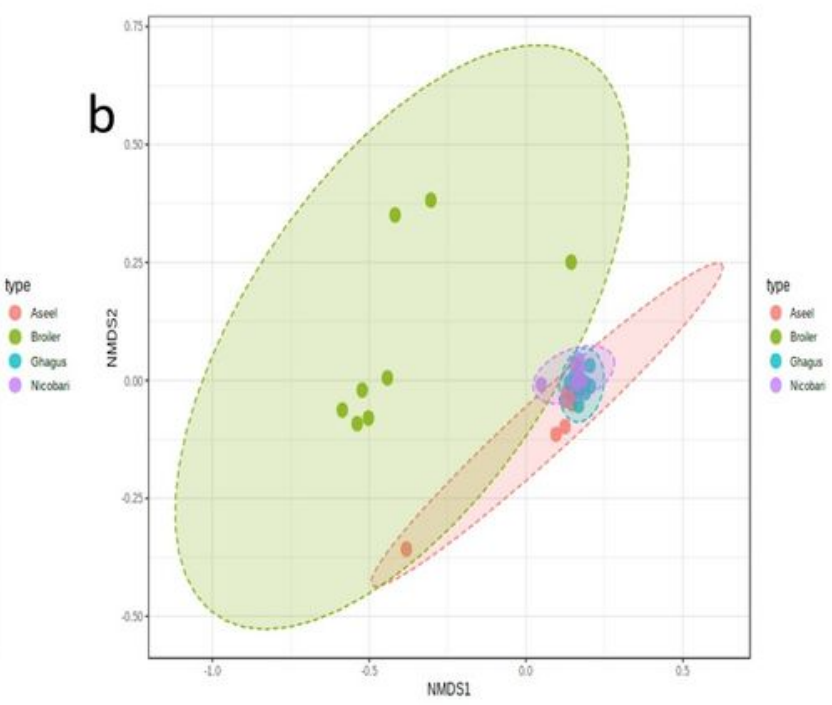

NMDS_Bray-Curtis_OTU;

Stress $=0.086253$

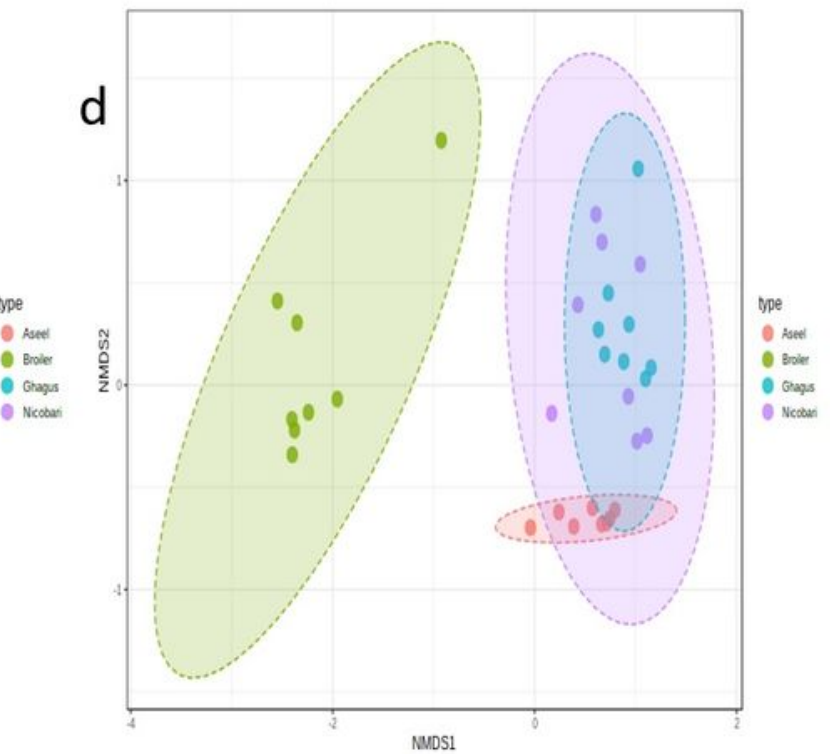

\section{Figure 3}

Beta diversity among breeds or line. Beta diversity plots visualized using Nonmetric multidimentional scaling based ordination at OTU level for different beta diversity metrices (a) Jensen-Shannon, (b) Weighted UniFrac, (c) Unweighted UniFrac, (d) Bray-Curtis. A stress value of less than 0.1 represents a high quality ordination. 


\section{NMDS_Jensen-Shannon_OTU; \\ Stress $=0.091409$}

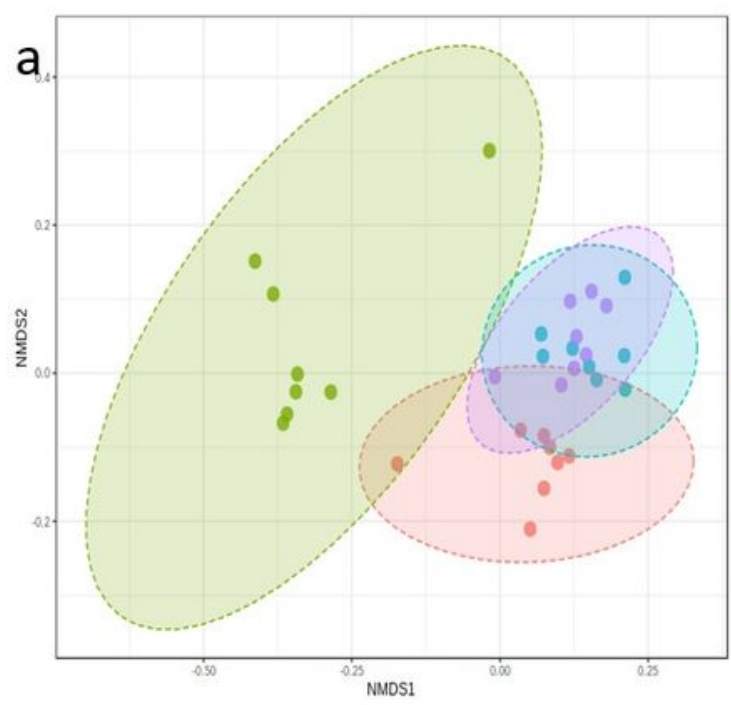

NMDS_unweighted UniFrac_ OTU; Stress $=0.069008$

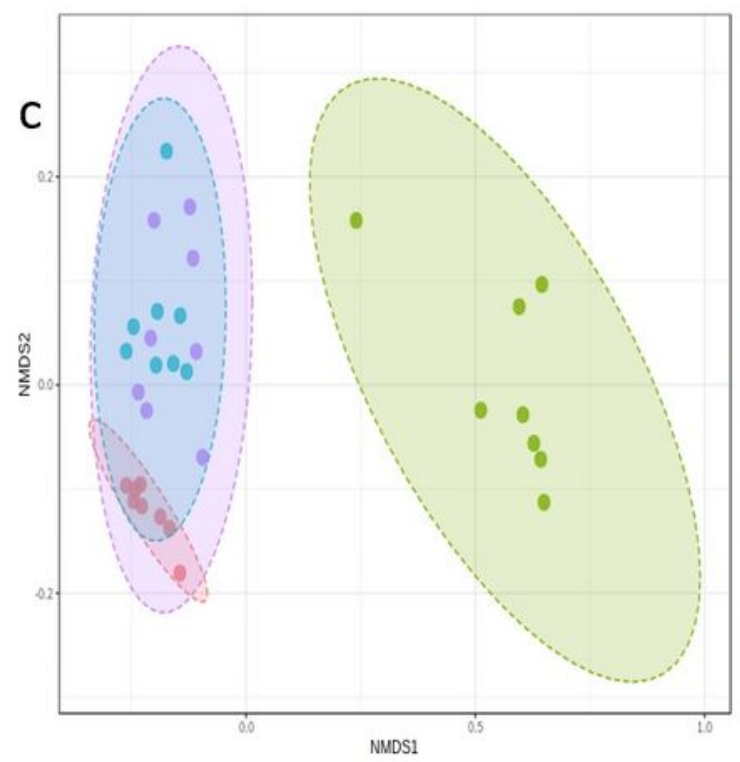

NMDS_Weighted UniFrac OTU;

Stress $=0.062276$

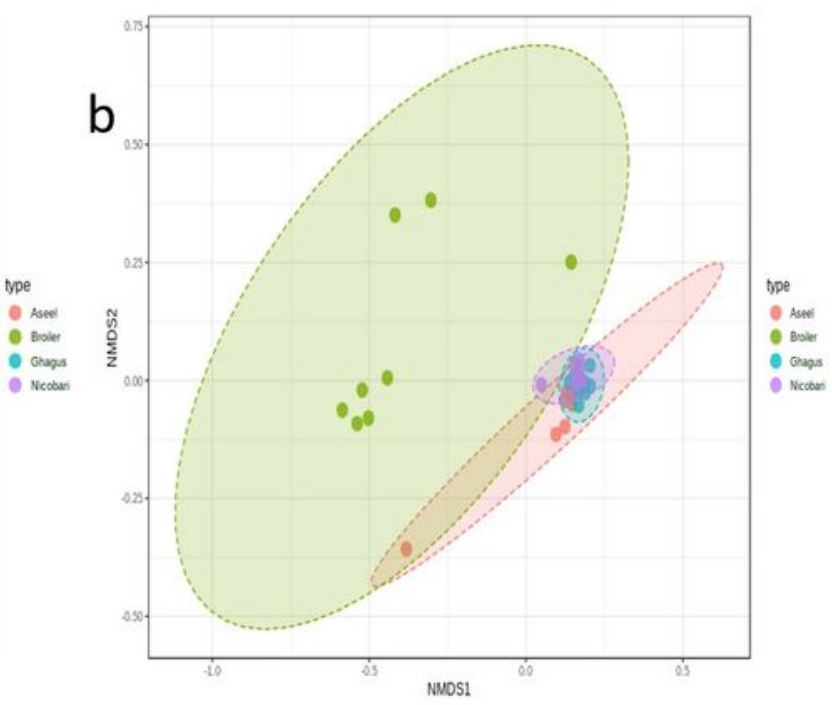

NMDS_Bray-Curtis_OTU;

Stress $=0.086253$

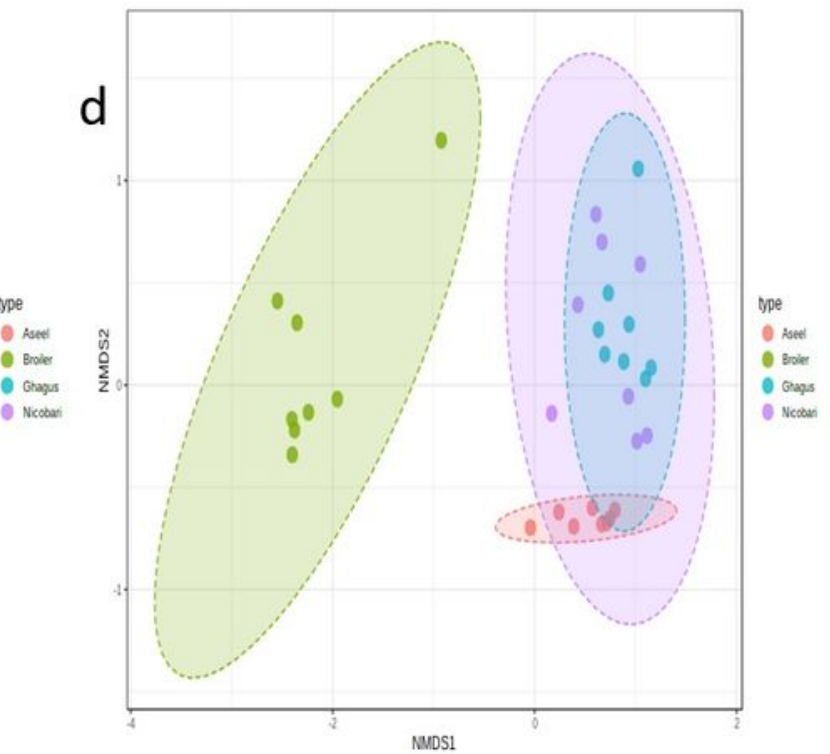

Figure 3

Beta diversity among breeds or line. Beta diversity plots visualized using Nonmetric multidimentional scaling based ordination at OTU level for different beta diversity metrices (a) Jensen-Shannon, (b) Weighted UniFrac, (c) Unweighted UniFrac, (d) Bray-Curtis. A stress value of less than 0.1 represents a high quality ordination. 


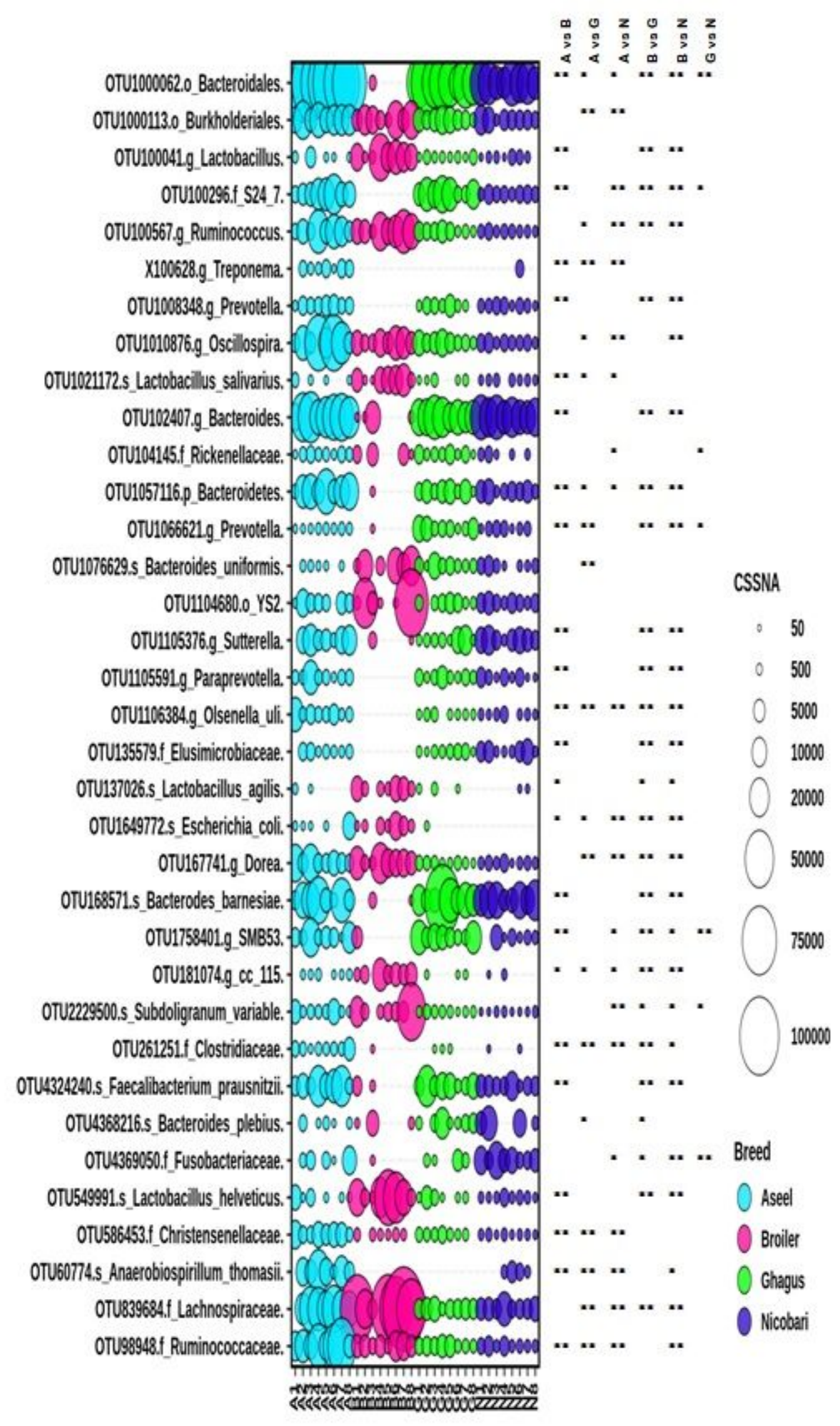

\section{Figure 4}

Differential abundance of gut microbiota in different breeds or line at OTU level. Top 35 abundant phylotype-OTUs out of 88 phylotype-OTUs with significant difference in relative abundance among groups identified with edgeR were plotted. The size of bubbles in the bubble plot indicates normalized (cumulative sum scaling) abundance of each OTU. 


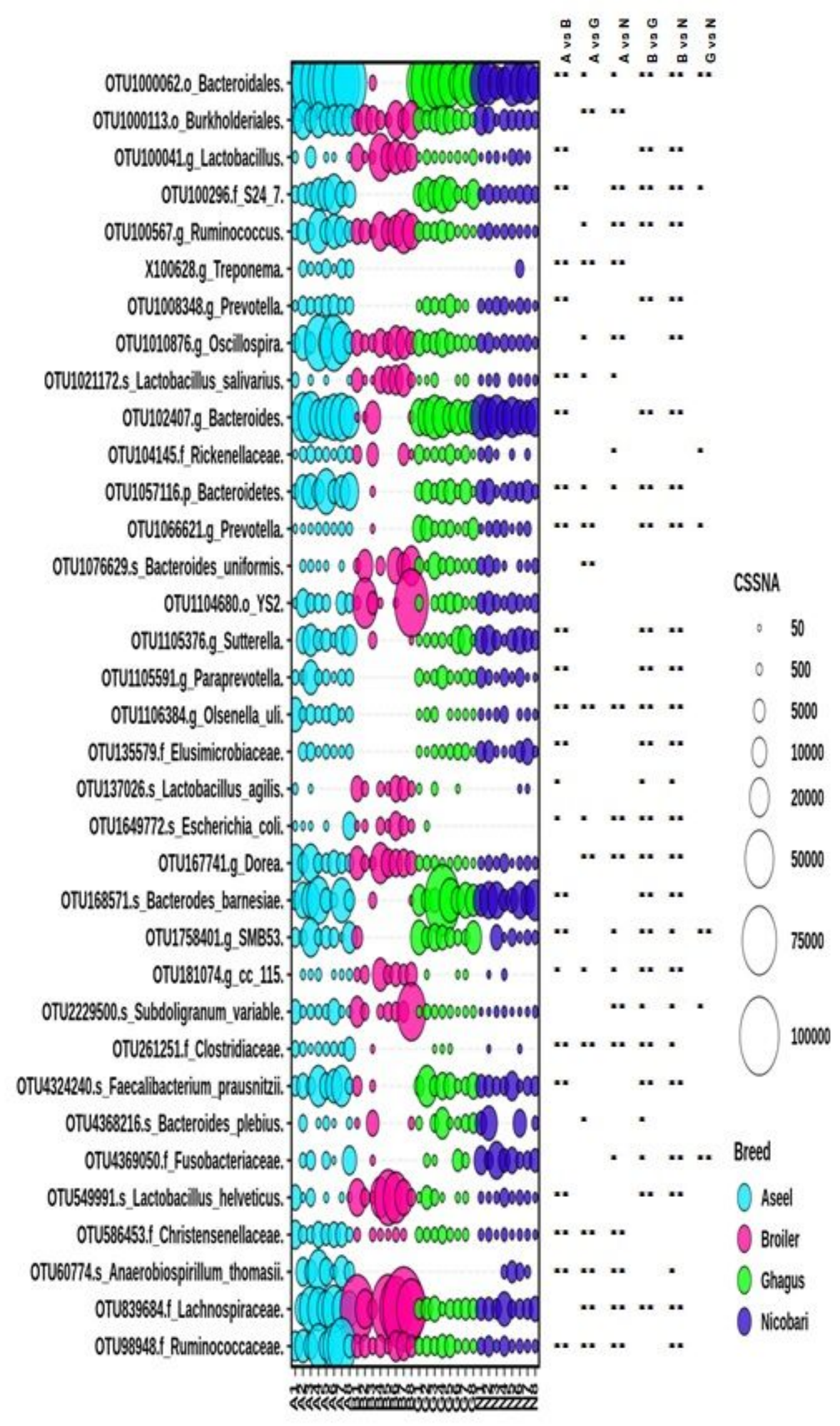

\section{Figure 4}

Differential abundance of gut microbiota in different breeds or line at OTU level. Top 35 abundant phylotype-OTUs out of 88 phylotype-OTUs with significant difference in relative abundance among groups identified with edgeR were plotted. The size of bubbles in the bubble plot indicates normalized (cumulative sum scaling) abundance of each OTU. 


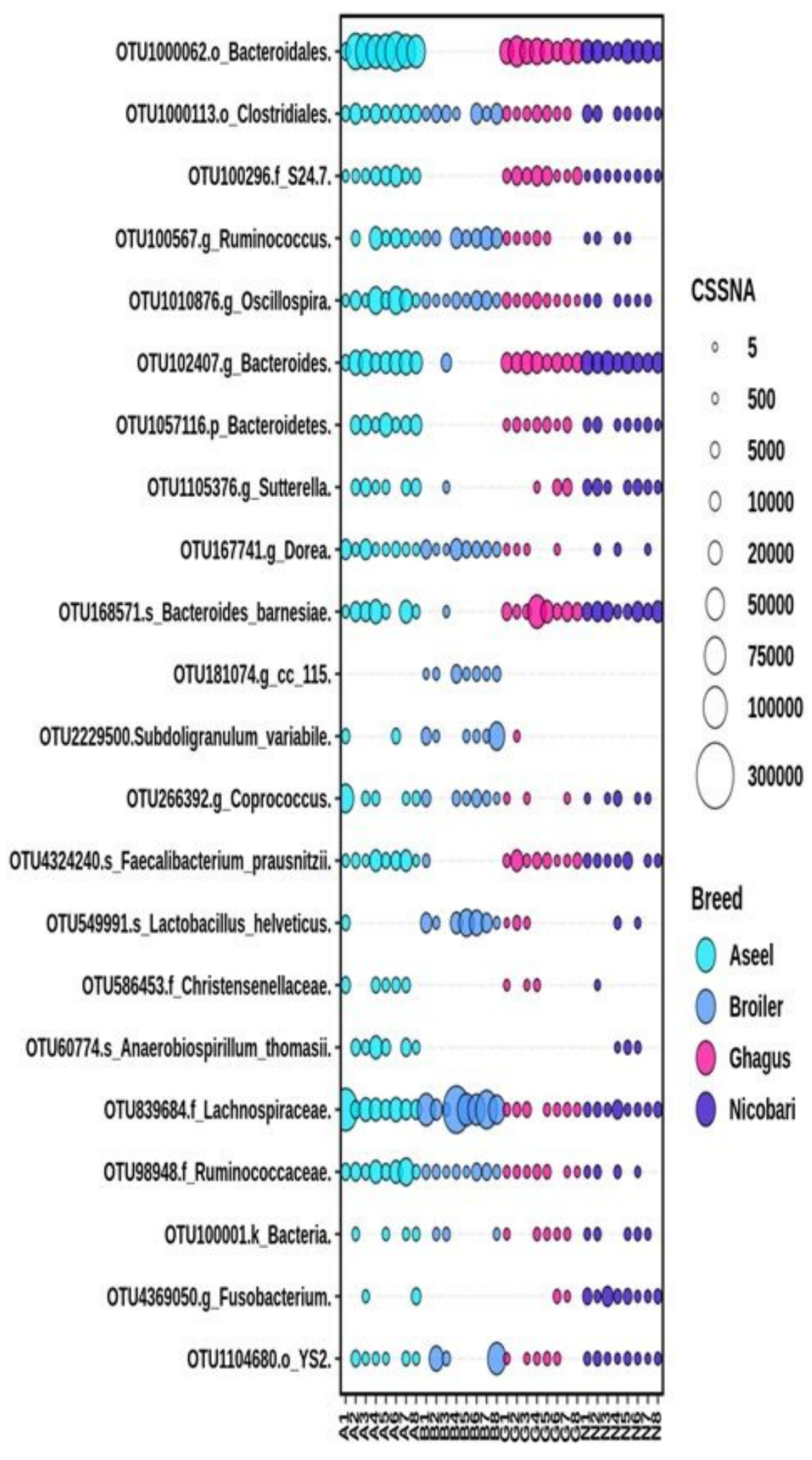

\section{Figure 5}

Breed or line specific and across-breed or line core phylotype-OTUs. Phylotype-OTUs with a mean normalized (cumulative sum scaling) relative abundance of at least $0.1 \%$ and having within breed or prevalence of at least $50 \%$ in at least one breed were taken into account The size of bubbles in the bubble plot indicates normalized (cumulative sum scaling) abundance of each OTU. 


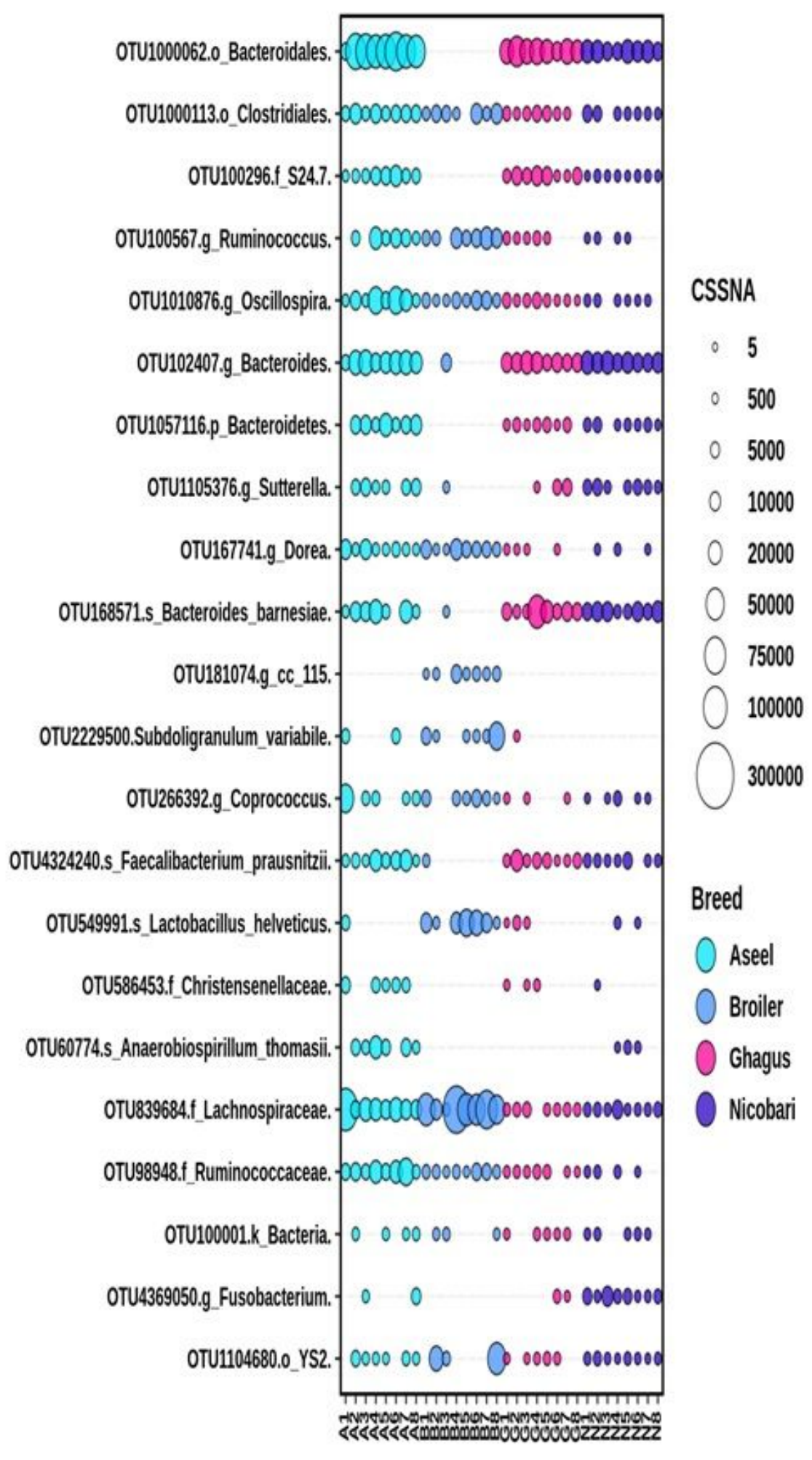

\section{Figure 5}

Breed or line specific and across-breed or line core phylotype-OTUs. Phylotype-OTUs with a mean normalized (cumulative sum scaling) relative abundance of at least $0.1 \%$ and having within breed or prevalence of at least $50 \%$ in at least one breed were taken into account The size of bubbles in the bubble plot indicates normalized (cumulative sum scaling) abundance of each OTU. 

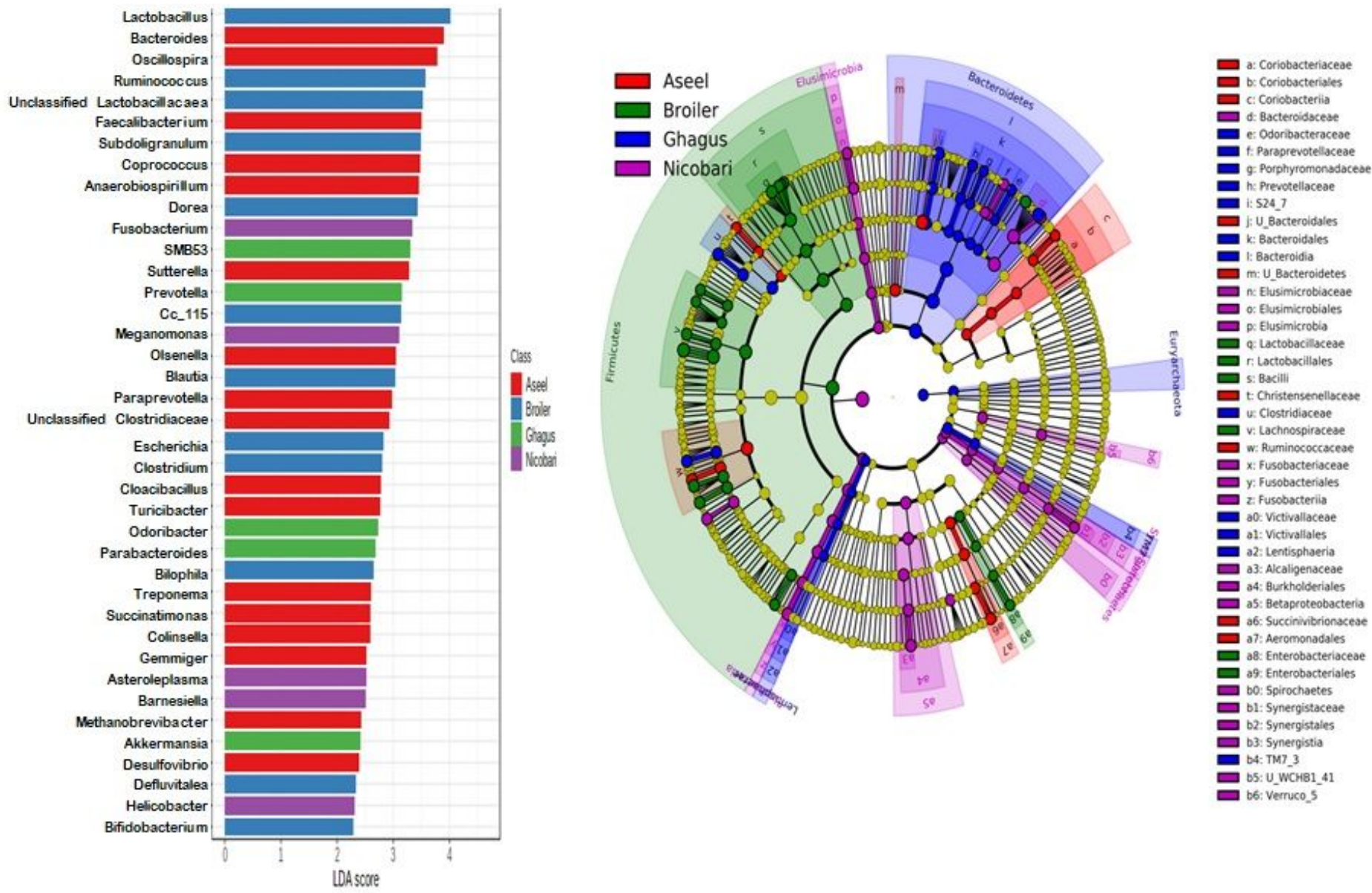

Figure 6

Chicken breed or line specific biomarkers. (a) Genus level biomarkers identified using LEfSe analysis using Kruskal-Wallis test $(P<0.05)$ with LDA score $>2.0$. (b) Cladogram representation of differentially abundant microbiota at different taxonomic level. The taxonomic levels of phylum is labeled, while order to genus are abbreviated (only labels of top 43 clades are shown here), with the colours indicating breed/line with the highest abundance. The cladogram has been dual rooted to denote domain archaea and bacteria. 

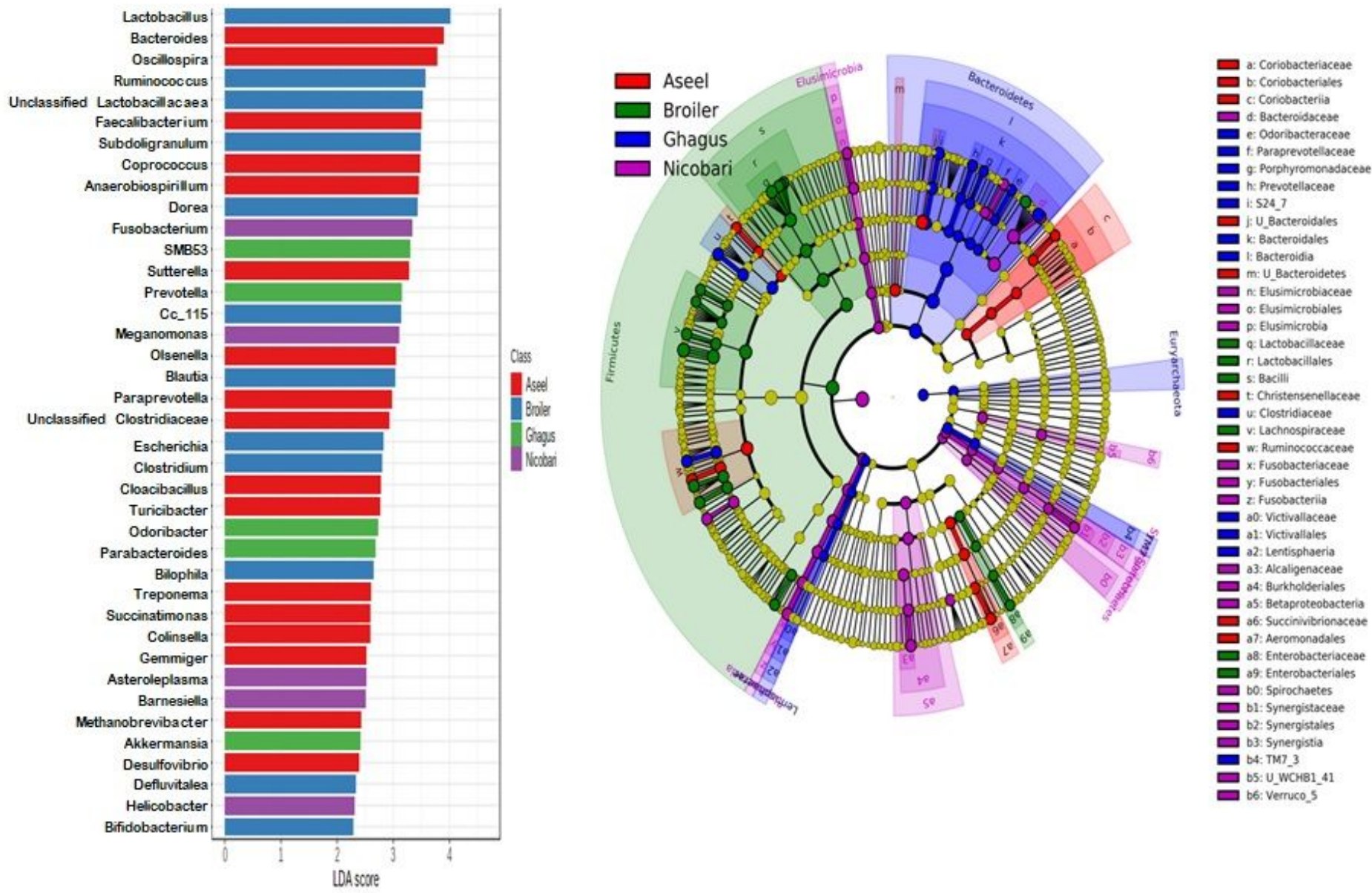

Figure 6

Chicken breed or line specific biomarkers. (a) Genus level biomarkers identified using LEfSe analysis using Kruskal-Wallis test $(P<0.05)$ with LDA score $>2.0$. (b) Cladogram representation of differentially abundant microbiota at different taxonomic level. The taxonomic levels of phylum is labeled, while order to genus are abbreviated (only labels of top 43 clades are shown here), with the colours indicating breed/line with the highest abundance. The cladogram has been dual rooted to denote domain archaea and bacteria. 


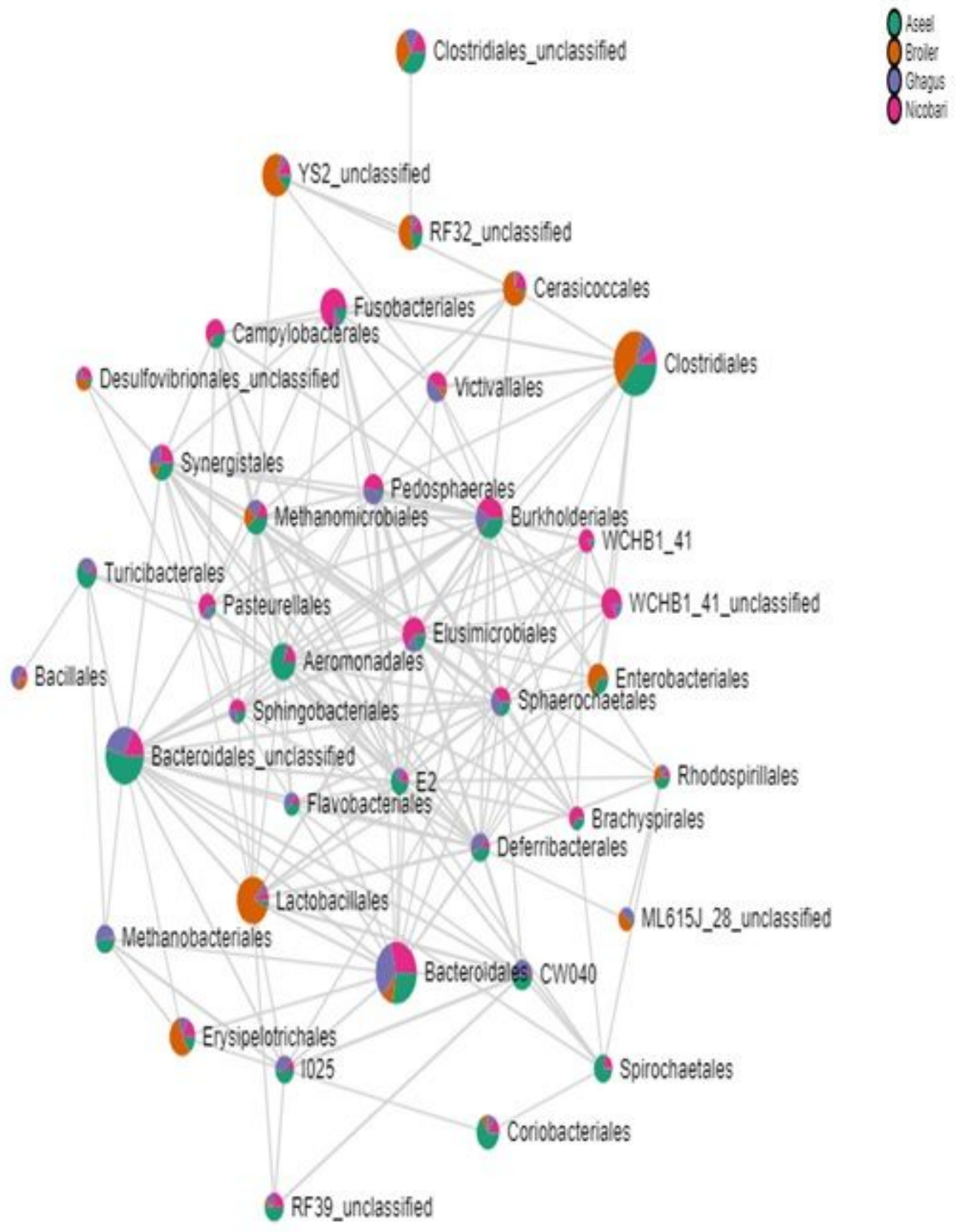

\section{Figure 7}

The genus and order level correlation networks have been presented in Additional file 15 and Figure 7 


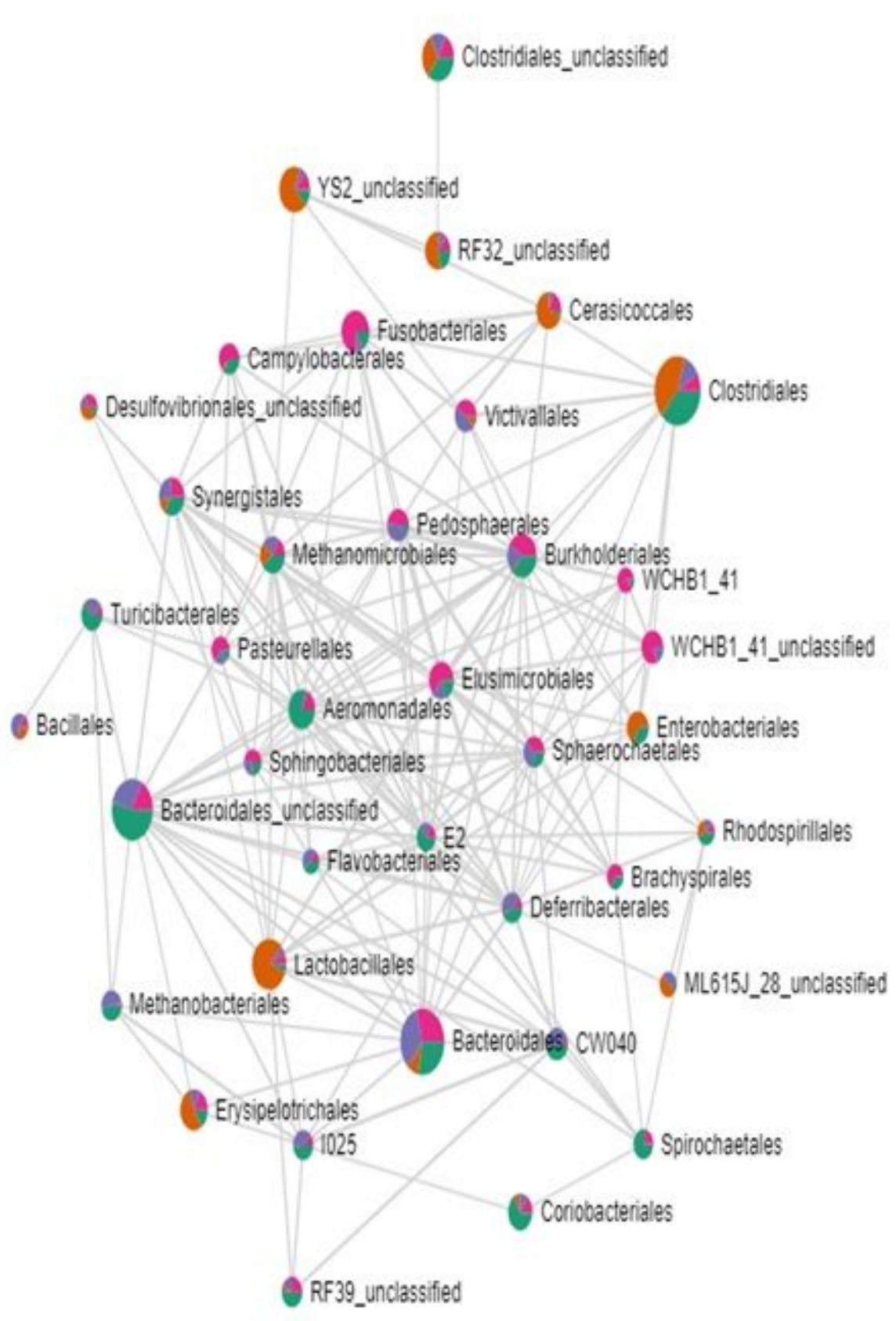

Figure 7

The genus and order level correlation networks have been presented in Additional file 15 and Figure 7

\section{Supplementary Files}

This is a list of supplementary files associated with this preprint. Click to download.

- Additionalfile5.pptx

- Additionalfile5.pptx 
- Additionalfile1.pptx

- Additionalfile1.pptx

- Additionalfile10.pptx

- Additionalfile10.pptx

- Additionalfile11.png

- Additionalfile11.png

- Additionalfile12.png

- Additionalfile12.png

- Additionalfile13.png

- Additionalfile13.png

- Additionalfile14.png

- Additionalfile14.png

- Additionalfile15.pptx

- Additionalfile15.pptx

- Additionalfile2.docx

- Additionalfile2.docx

- Additionalfile3.pptx

- Additionalfile3.pptx

- Additionalfile4.docx

- Additionalfile4.docx

- Additionalfile6.pptx

- Additionalfile6.pptx

- Additionalfile7.pptx

- Additionalfile7.pptx

- Additionalfile8.pptx

- Additionalfile8.pptx

- Additionalfile9.pptx

- Additionalfile9.pptx 